\title{
A hybrid numerical approach to predict the vibrational responses of panels excited by a turbulent boundary layer
}

\author{
M. Karimi ${ }^{*}$, P. Croaker ${ }^{2}$, L. Maxit ${ }^{3}$, O. Robin ${ }^{4}$, \\ A. Skvortsov 5 , S. Marburg 6 , N. Kessissoglou ${ }^{2}$ \\ 1 Centre for Audio, Acoustics and Vibration, University of Technology Sydney, Sydney, \\ Australia \\ 2 School of Mechanical and Manufacturing Engineering, UNSW Sydney, Australia \\ 3 Univ Lyon, INSALyon, Laboratoire Vibrations-Acoustique (LVA), 25 bis, av. Jean \\ Capelle, F-69621, Villeurbanne Cedex, France \\ 4 Groupe d'Acoustique de l'Université de Sherbrooke, Université de Sherbrooke, \\ Sherbrooke, J1K 2R1, Canada \\ 5 Maritime Division, Defence Science and Technology, Melbourne, Australia \\ 6 Chair of Vibroacoustics of Vehicles and Machines, Department of Mechanical \\ Engineering, Technische Universität München, München, Germany
}

\begin{abstract}
In this work, a hybrid numerical approach to predict the vibrational responses of planar structures excited by a turbulent boundary layer is presented. The approach combines an uncorrelated wall plane wave technique with the finite element method. The wall pressure field induced by a turbulent boundary layer is obtained as a set of uncorrelated wall pressure plane waves. The amplitude of these plane waves are determined from the cross spectrum density function of the wall pressure field given either by empirical models from literature or from experimental data. The response of the planar structure subject to a turbulent boundary layer excitation is then obtained from an ensemble average of the different realizations. The numerical technique is computationally efficient as it rapidly converges using a small number of realizations. To demonstrate the method, the vibrational responses of two panels with simply supported or clamped boundary conditions and excited by turbulent flow are considered. In the case study comprising a plate with
\end{abstract}

\footnotetext{
*Corresponding author

Email address: Mahmoud.Karimi@uts.edu.au
} 
simply supported boundary conditions, an analytical solution is employed for verification of the method. For both cases studies, numerical results from the hybrid approach are compared with experimental data measured in two different anechoic wind tunnels.

Keywords: Uncorrelated wall plane wave, finite element method, turbulent boundary layer, flow-induced vibration

\section{INTRODUCTION}

The vibrational response of elastic structures subject to flow excitation is a seminal problem in a variety of technical applications, for example, an aircraft fuselage excited by turbulent boundary layer, a hydrofoil operating in turbulent flow or a telecommunications tower excited by wind. In such cases, the correct prediction of the vibrational response is crucial to minimise structural fatigue as well as structure-borne radiating noise (Leibowitz, 1975; Boily and Charron, 1999; Ciappi et al., 2014, 2018). To predict the vibrational responses for such problems, the forcing function arising from the turbulent flow field on the elastic structure should be obtained. This can be achieved by solving the Navier Stokes equations for given geometry and flow conditions. Numerical approaches such as direct numerical simulation (DNS) or large eddy simulation (LES) can be implemented to solve the Navier Stokes equations as well as take into account interaction of the flow with the body. However, these methods are computationally demanding as their implementation for simulation of realistic scenarios often become impractical due to the significant range of spatial and temporal scales of the turbulence that need to be resolved (Shtilman and Chasnov, 1992). An alternative approach involves a steady-state Reynolds-averaged Navier Stokes (RANS) solution to predict the turbulent boundary layer (TBL) parameters (Bailly et al., 1997; Peltier and Hambric, 2007). RANS is an attractive approach as it is capable of predicting TBL parameter mean values with good fidelity. These parameters can then be used as an input to analytical or semi-empirical models to predict the wall pressure field under the turbulent boundary layer (Blake, 1986; Lee et al., 2005; Chen and MacGillivray, 2014).

A vast number of studies have been carried out to predict the vibrational responses of plates excited by a turbulent flow field in air, including analytical models of infinite and finite plates (Strawderman, 1969; De Rosa and Franco, 2008), numerical models (Birgersson et al., 2003; Hambric et al., 2004; Birg- 
ersson and Finnveden, 2005; De Rosa and Franco, 2008; Hong and Shin, 2010; Ciappi et al., 2016), and from experiments (Ciappi et al., 2016). Birgersson et al. (2003) proposed a wavenumber domain approach to investigate the response of structures to TBL excitation. The cross spectral density of the TBL wall pressure was expressed as a finite Fourier series. The structural response to each term in the series was calculated using the spectral finite element method (FEM) and the total response was then obtained using the superposition principle. A spectral super element formulation for modelling plate vibration excited by distributed forces was developed by Birgersson and Finnveden (2005). Ichchou et al. (2009) employed "rain-on-the-roof" excitation represented by statistically independent point sources, to excite a flat plate in the mid frequency range, showing good agreement with results obtained using an FEM model. Turbulence induced vibration of aerospace composite plates was numerically and experimentally investigated by Ciappi et al. (2016). It was demonstrated that at high Mach number, the fluidloading effect on the composite panels cannot be neglected. More recently, Marchetto et al. (2017) compared the responses of a panel excited by a TBL as well as by a diffuse acoustic field.

De Rosa et al. (2015) presented a pseudo deterministic excitation method to evaluate the dynamic response of a linear system excited by a turbulent load. The method achieved a significant reduction in computational time compared to the full stochastic solution. Errico et al. (2018) proposed an approach to model structures excited by aerodynamic and acoustic sources based on a wave finite element method. The approach was employed to predict flow-induced vibrations of periodic flat and curved structures (Errico et al., 2019). Similitude laws for scaling the vibration response of flat panels to a turbulent boundary layer excitation were proposed by Franco et al. (2019). These laws remove the necessity of repeating experiment or numerical simulations due to the change in flow speeds, dimensions and material properties of panel.

In many numerical approaches to predict vibrational responses of structures excited by turbulent flow, the system response depends on the cross spectrum density (CSD) function of the wall pressure fluctuations. In order to correctly describe the partial correlation of the excitation, a large number of points distributed on the structural surface from which the frequency response functions are calculated need to be considered (Hambric et al., 2004; Hong and Shin, 2010). The coupling between a statistical model to describe the wall pressure fluctuations and a deterministic numerical model of the 
structure represents a difficulty in the calculation process. The current study proposes a way of addressing this difficulty by employing a hybrid numerical approach involving the coupling between statistical and deterministic methods, to predict the vibrational response of a panel under TBL excitation. To this end, the turbulent boundary layer excitation is modelled using a set of uncorrelated wall plane waves. The synthesized wall pressure corresponding to each realization within the set of uncorrelated wall plane waves is applied to a standard FEM model of the panel. This process is repeated for each realization of the wall pressure field. The vibrational response of the panel is then obtained from an ensemble average of the different realizations of the wall pressure. To demonstrate the technique, two case studies are considered corresponding to simply supported and clamped plates. The vibrational response is predicted numerically and analytically (for the simply supported plate). The results for both case studies are validated with experimental data.

\section{Mathematical formulation}

\subsection{Hybrid numerical approach}

Figure 1 shows an elastic rectangular baffled panel with arbitrary boundary conditions. The plate is excited by a turbulent flow field. It is assumed that the TBL is homogeneous, stationary and fully developed over the panel surface. Further, it is assumed that the wall pressure field (WPF) is not altered by the vibration of the panel.

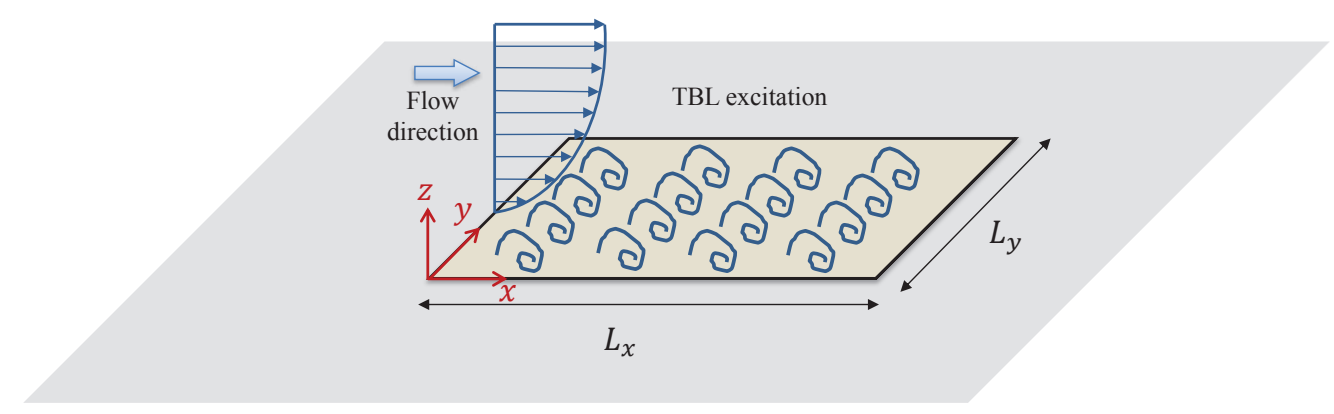

Figure 1: A baffled panel under TBL excitation

The uncorrelated wall plane wave technique (UWPW) recently introduced by Maxit (2016) is used to simulate the pressure field beneath a turbulent 
boundary layer. The UWPW approach is summarized in what follows. The space-frequency cross spectrum of the wall pressure fluctuations is given by (Graham, 1997; Maxit, 2016)

$$
S_{p p}^{\mathrm{TBL}}\left(\mathbf{x}-\mathbf{x}^{\prime}, \omega\right)=\Psi_{p p}(\omega)\left(\frac{U_{c}}{\omega}\right)^{2} \tilde{S}_{p p}\left(\mathbf{x}-\mathbf{x}^{\prime}, \omega\right)
$$

where $\Psi_{p p}(\omega)$ and $\tilde{S}_{p p}\left(\mathbf{x}-\mathbf{x}^{\prime}, \omega\right)$ are respectively the auto spectrum density (ASD) function and normalized CSD function of the pressure field. $U_{c}$ is the convective velocity, $\omega$ is the angular frequency, and $\mathbf{x}, \mathbf{x}^{\prime}$ correspond to point locations on the plate. The CSD of the wall pressure field in the physical space is related to the CSD of the wall pressure spectrum in the wavenumber domain, denoted by $\phi_{p p}(\mathbf{k}, \omega)$, using a spatial Fourier transform as follows

$$
S_{p p}^{\mathrm{TBL}}\left(\mathbf{x}-\mathbf{x}^{\prime}, \omega\right)=\frac{1}{4 \pi^{2}} \int_{\infty} \phi_{p p}(\mathbf{k}, \omega) e^{\mathrm{i} \mathbf{k}\left(\mathbf{x}-\mathbf{x}^{\prime}\right)} d \mathbf{k},
$$

where $\mathrm{i}=\sqrt{-1}$ is the imaginary unit and $\mathbf{k}$ is the wavevector with components $k_{x}$ and $k_{y}$ in the streamwise and spanwise directions, respectively. The cross spectrum of the wall pressure can be computed using different models for the ASD of the pressure field and the normalized CSD of the pressure field, denoted by $\tilde{\phi}_{p p}(\mathbf{k}, \omega)$, independently from each other as follows

$$
\phi_{p p}(\mathbf{k}, \omega)=\Psi_{p p}(\omega)\left(\frac{U_{c}}{\omega}\right)^{2} \tilde{\phi}_{p p}(\mathbf{k}, \omega) .
$$

The improper integral in equation (2) can be approximated using the rectangular method by truncating and sampling the wavenumber space as follows (Maxit, 2016)

$$
S_{p p}^{\mathrm{TBL}}\left(\mathbf{x}-\mathbf{x}^{\prime}, \omega\right) \approx \frac{1}{4 \pi^{2}} \sum_{i=1}^{N_{x}} \sum_{j=1}^{N_{y}} \phi_{p p}\left(k_{x}^{i}, k_{y}^{j}, \omega\right) e^{\mathrm{ik}\left(\mathbf{x}-\mathbf{x}^{\prime}\right)} \delta k_{x} \delta k_{y} .
$$

where $\delta k_{x}, \delta k_{y}$ are the wavenumber resolutions in the streamwise and spanwise directions, respectively. $N_{x}$ and $N_{y}$ are the number of points considered along the $k_{x}$ and $k_{y}$ directions. The total pressure beneath a turbulent boundary layer is now represented by a set of UWPWs. As the wall plane waves 
are uncorrelated, the CSD function of the wall pressure fluctuations induced by a set of wall plane waves can be written as

$$
S_{p p}^{\mathrm{UWPW}}\left(\mathbf{x}-\mathbf{x}^{\prime}, \omega\right)=\sum_{i=1}^{N_{x}} \sum_{j=1}^{N_{y}} \Lambda_{i j} e^{\mathrm{ik}\left(\mathbf{x}-\mathbf{x}^{\prime}\right)},
$$

where $\Lambda_{i j}$ is the ASD function of the stochastic amplitude of UWPW with indices $i$ and $j$. Equating equations (4) and (5) allows the CSD function of the pressure field by the TBL to be approximately equal to the CSD function of the UWPWs if the amplitudes of the UWPWs are

$$
\Lambda_{i j}=\frac{\phi_{p p}\left(k_{x}, k_{y}, \omega\right) \delta k_{x} \delta k_{y}}{4 \pi^{2}} .
$$

The amplitude of each wall pressure plane wave is defined such that the set of UWPWs represent the statistical properties of the WPF generated by the TBL. The statistical model to describe the WPF can now be coupled to a deterministic model based on the FEM. This important step allows the WPF to be expressed as a deterministic load input to the FEM. The WPF for the $l^{\text {th }}$ realization of the UWPW can be expressed by (Maxit, 2016; Karimi et al., 2019)

$$
p_{\text {inc }}^{l}(\mathbf{x}, \omega)=\sum_{i=1}^{N_{x}} \sum_{j=1}^{N_{y}} \sqrt{\Lambda_{i j}} e^{\mathrm{i}\left(k_{x} x+k_{y} y+\varphi_{i j}^{l}\right)},
$$

where $\varphi$ is a random phase uniformly distributed in $\left[\begin{array}{ll}0 & 2 \pi\end{array}\right]$, expressing that the waves are uncorrelated. Substituting equation (6) into equation (7) and considering the $q^{\text {th }}$ node of an FEM mesh, the nodal pressure can be written as follows

$$
p_{\text {inc }}^{l}\left(\mathbf{x}^{q}, \omega\right)=\sum_{i=1}^{N_{x}} \sum_{j=1}^{N_{y}} \sqrt{\frac{\phi_{p p}\left(k_{x}, k_{y}, \omega\right) \delta k_{x} \delta k_{y}}{4 \pi^{2}}} e^{\mathrm{i}\left(k_{x} x^{q}+k_{y} y^{q}+\varphi_{i j}^{l}\right)} .
$$

Using equation (8) as the deterministic load, FEM is now implemented to compute the $l^{\text {th }}$ realization of the structural displacement $\mathbf{u}^{l}$ by solving the following linear system of equations

$$
\mathrm{Du}^{l}=\mathbf{f}^{l}
$$

where $\mathbf{D}$ is the dynamic stiffness given by

$$
\mathbf{D}=\mathbf{K}-\mathrm{i} \omega \mathbf{C}-\omega^{2} \mathbf{M}
$$


$\mathbf{K}, \mathbf{C}$ and $\mathbf{M}$ are respectively stiffness, damping and mass matrices of the structure, and $\mathbf{f}^{l}$ is the force vector corresponding to the $l^{\text {th }}$ realization of the TBL pressure field given by equation (8). It should be noted that the mass and stiffness matrices are not frequency dependent and need to be constructed only once for a given geometry. After the inverse of the dynamic stiffness matrix is obtained, the plate displacement response can be computed for each realization as follows

$$
\mathbf{u}^{l}=\mathbf{D}^{-1} \mathbf{f}^{l} .
$$

The ASD of the plate displacement due to the TBL excitation is then calculated from the ensemble average of the different realizations by

$$
S_{u u}=\mathrm{E}\left[\mathbf{u}^{l} \overline{\mathbf{u}}^{l}\right]_{l},
$$

where $\mathrm{E}[$ ] represents the ensemble average over the realizations and the overline denotes the complex conjugate. This process is repeated for each frequency to obtain the spectra of the structural response.

Figure 2 illustrates the computational sequences for the UWPW-FEM approach. First, a mesh is created from the geometry. A RANS simulation, theoretical formula or experimental data can be used to estimate the TBL parameters over the surface of a structure for a given geometry and flow condition. The cross-spectrum of the wall pressure is evaluated from the TBL parameters using semi-empirical models. The spectra of the wall pressure is then applied in conjunction with the UWPW technique to obtain the WPF. The WPF is then used as an input to the FEM solver to compute the vibrational response. This process is repeated for each realization of the WPF. Finally, the structural response of the system is obtained from an ensemble average of the different realizations of the wall pressure fields at each frequency.

\subsection{Analytical model}

For verification of the hybrid numerical approach, an analytical model is herein presented for the case of a plate with simply supported boundary conditions on all its edges. The vibrational response of a panel due to excitation by a pressure field can be obtained using the modal expansion method and by identifying a panel sensitivity function. The ASD of the panel velocity is 


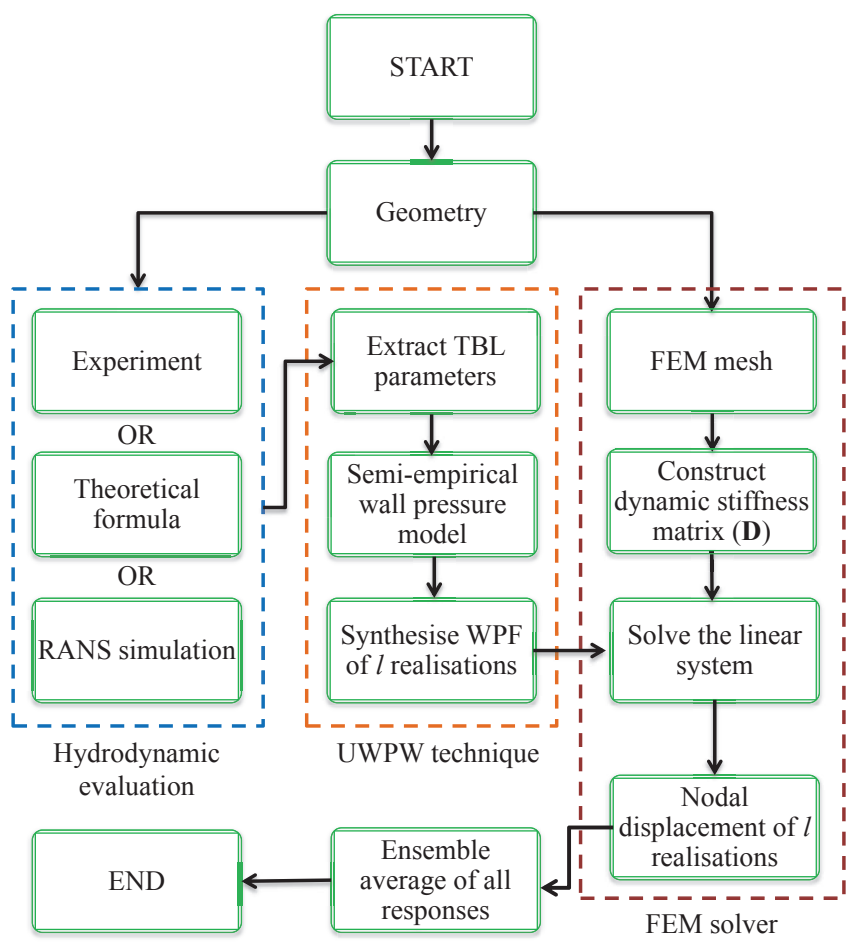

Figure 2: Flowchart outlining the computational process of the hybrid UWPW-FEM approach

given by (Maury et al., 2002)

$$
S_{v v}(\mathbf{x}, \omega)=\frac{1}{4 \pi^{2}} \int_{\infty} \phi_{p p}(\mathbf{k}, \omega)|H(\mathbf{x}, \mathbf{k}, \omega)|^{2} d \mathbf{k},
$$

121

where $H(\mathbf{x}, \mathbf{k}, \omega)$ is the sensitivity function which corresponds to the velocity at point $\mathbf{x}$ when the panel is excited by a unit wall plane wave. $\phi_{p p}(\mathbf{k}, \omega)$ is the CSD of the wall pressure field defined previously. Using numerical integration based on the rectangular method by truncating and regularly sampling the wavenumber domain, equation (13) can be expressed as

$$
S_{v v}(\mathbf{x}, \omega) \approx \frac{1}{4 \pi^{2}} \sum_{i=1}^{N_{x}} \sum_{j=1}^{N_{y}} \phi_{p p}(\mathbf{k}, \omega)|H(\mathbf{x}, \mathbf{k}, \omega)|^{2} \delta k_{x} \delta k_{y},
$$

Using the normal modes of the panel, an analytical solution for the panel 
sensitivity function can be derived as follows (Maxit, 2016; Marchetto et al., 2017)

$$
H(\mathbf{x}, \mathbf{k}, \omega)=\mathrm{i} \omega \sum_{m=1}^{M} \sum_{n=1}^{N} \frac{\psi_{m n}(\mathbf{k}) \varphi_{m n}(\mathbf{x})}{\Omega_{m n}\left(\omega_{m n}^{2}-\omega^{2}+\mathrm{i} \eta \omega \omega_{m n}\right)},
$$

where $M$ and $N$ are the truncation number of modal orders in the $x$ and $y$ directions, respectively. For the modal expansion, the number of modes within the extended frequency band $\left[\begin{array}{ll}0 & 1.5 \omega_{\max }\end{array}\right]$ were considered, where $\omega_{\max }$ represents the highest angular frequency of interest. $\eta$ is the structural loss factor, $\Omega_{m n}=\rho_{s} h L_{x} L_{y} / 4$ is the modal mass, $\omega_{m n}$ and $\varphi_{m n}$ are respectively the modal frequencies and mode shapes of the panel given by

$$
\begin{gathered}
\omega_{m n}=\sqrt{\frac{D}{\rho_{s} h}}\left(\left(\frac{m \pi}{L_{x}}\right)^{2}+\left(\frac{n \pi}{L_{y}}\right)^{2}\right), \\
\varphi_{m n}(\mathbf{x})=\sin \left(\frac{m \pi x}{L_{x}}\right) \sin \left(\frac{n \pi y}{L_{y}}\right) .
\end{gathered}
$$

$L_{x}$ and $L_{y}$ are respectively the length and width of the plate. $D=E h^{3} /(12(1-$ $\left.\nu^{2}\right)$ ) is the flexural rigidity, $\rho_{s}$ is the density, $h$ is the panel thickness, $E$ is the Young's modulus and $\nu$ is Poisson's ratio. The modal forces $\psi_{m n}$ are calculated by integration over the panel surface $A$ as follows

$$
\psi_{m n}\left(k_{x}, k_{y}\right)=\int_{A} e^{\mathrm{i}\left(k_{x} x+k_{y} y\right)} \varphi_{m n}(\mathbf{x}) d A=I_{m}^{x} I_{n}^{y}
$$

where

$$
\left\{I_{s}^{r} \mid(r, s)=(x, m) \vee(y, n)\right\}=\left\{\begin{array}{ll}
\left(\frac{s \pi}{L_{r}}\right) \frac{(-1)^{s} e^{\mathrm{i}\left(k_{r} L_{r}\right)}-1}{k_{r}^{2}-\left(\frac{s \pi}{L_{r}}\right)^{2},} & k_{r} \neq \frac{s \pi}{L_{r}} \\
\frac{1}{2} \mathrm{i} L_{r}, & \text { otherwise }
\end{array}\right\} .
$$

\section{Results and discussion}

To demonstrate the UWPW-FEM approach, two case studies comprising rectangular panels with different boundary conditions and excited by a TBL are examined. The first case study investigates a panel with simply supported boundary conditions. Numerical results for the simply supported 
panel from the UWPW-FEM technique as well as analytical results obtained using the sensitivity function are compared with experimental data obtained in an anechoic wind tunnel. The second case study examines the vibrational response of a panel with clamped boundary conditions. Dimensions and material properties of both panels are given in Table 1 . The fluid density and the kinematic viscosity were set to $1.225 \mathrm{~kg} / \mathrm{m}^{3}$ and $1.5111 \times 10^{-5} \mathrm{~m}^{2} / \mathrm{s}$, respectively. For each case study, the structural loss factor was experimentally estimated using the $-3 \mathrm{~dB}$ bandwidth method for the first few resonances of the plate. The mean value of the loss factors was used in the numerical simulations. The simulations were conducted using Matlab on a desktop personal computer with 32 GB of RAM and a total of four physical cores. For the UWPW-FEM technique, the wall pressure field was synthesized in Matlab and then imported as a load to the FEM model of the panel in the commercial software COMSOL Multiphysics (v5.3a) using Matlab LiveLink.

To obtain the panel response analytically or numerically, truncation of wavenumber domain is required. A truncated number of wavenumbers in the $x$ and $y$ directions need to be defined for equations (8) and (14). The criterion for defining the cut-off wavenumbers in the streamwise and spanwise directions must be chosen such that the significant contributions of the integrands of these equations are correctly taken into account. It has been previously demonstrated that in the vibration response, the wavenumbers below or close to the natural flexural wavenumber of the plate are dominant (Hambric et al., 2004; Maxit, 2016; Marchetto et al., 2018). Hence, a cut-off wavenumber of $2 k_{p, \max }$ was used in both the streamwise and spanwise directions, where $k_{p, \max }=\left(\omega_{\max } \sqrt{\rho_{s} h / D}\right)^{1 / 2}$ is the flexural wavenumber of the plate at the maximum frequency of interest denoted by $\omega_{\max }$. The wavenumber resolutions were set to $\delta k_{x}=\delta k_{y}=0.25(1 / \mathrm{m})$. Based on the cut-off wavenumber, a mesh size of $\Delta x=\Delta y=\pi /\left(2 k_{p, \max }\right)$ was selected in this work. A frequency resolution of $2 \mathrm{~Hz}$ was used in all numerical simulations. It is worth noting that if a very wide frequency range is considered, the frequency range can be divided into frequency bands. A different mesh size based on the highest frequency of interest for a given band can be employed, thereby increasing the efficiency of the method.

\subsection{Case study A - Simply supported plate}

The first case study considers a simply supported baffled rectangular panel used in the experiment by Marchetto et al. (2018) which was conducted in an anechoic wind tunnel at the Université de Sherbrooke. The 
Table 1. Dimensions and mechanical properties of the panels

\begin{tabular}{lcc}
\hline Parameter & $\begin{array}{c}\text { Case A } \\
\text { supported plate }\end{array}$ & $\begin{array}{c}\text { Case B } \\
\text { Clamped plate }\end{array}$ \\
\hline Young's modulus $E(\mathrm{GPa})$ & 70 & 200 \\
Poisson's ratio $\nu$ & 0.3 & 0.29 \\
Density $\rho\left(\mathrm{kg} / \mathrm{m}^{3}\right)$ & 2700 & 7850 \\
Length $L_{x}(\mathrm{~mm})$ & 480 & 470 \\
Width $L_{y}(\mathrm{~mm})$ & 420 & 370 \\
Thickness $h(\mathrm{~mm})$ & 3.17 & 1.59 \\
Damping loss factor $\eta$ & 0.005 & 0.005 \\
\hline
\end{tabular}

experimental fabrication method proposed by Robin et al. (2016) was used to replicate simply supported boundary conditions. The panel was made of aluminium and the edge of the panel was placed $1.8 \mathrm{~m}$ from the nozzle. The vibration of the panel was measured using an accelerometer located at $(x=0.3 \mathrm{~m}, y=0.33 \mathrm{~m}, z=0 \mathrm{~m})$ on the panel surface as shown in Figure 3. The experiments were conducted at flow speeds of $20 \mathrm{~m} / \mathrm{s}$ and $40 \mathrm{~m} / \mathrm{s}$.

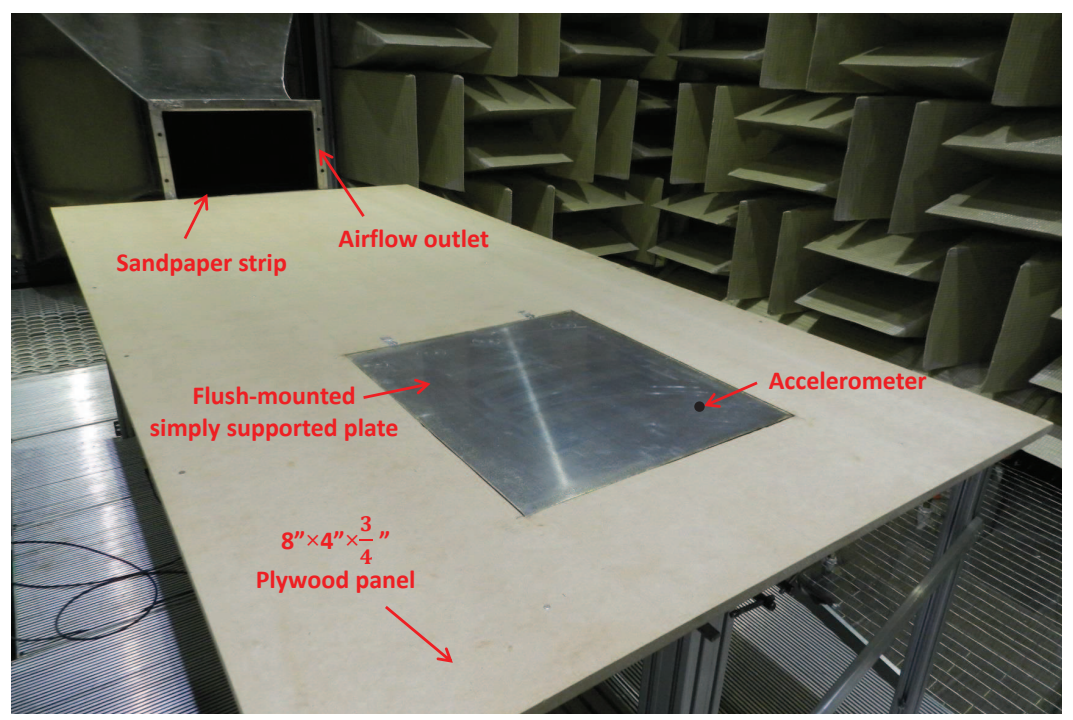

Figure 3: Experimental set-up in the anechoic wind tunnel at the Université de Sherbrooke (Marchetto et al., 2018). The accelerometer location is at $(x=0.3 \mathrm{~m}, y=0.33 \mathrm{~m}, z=0 \mathrm{~m})$ 


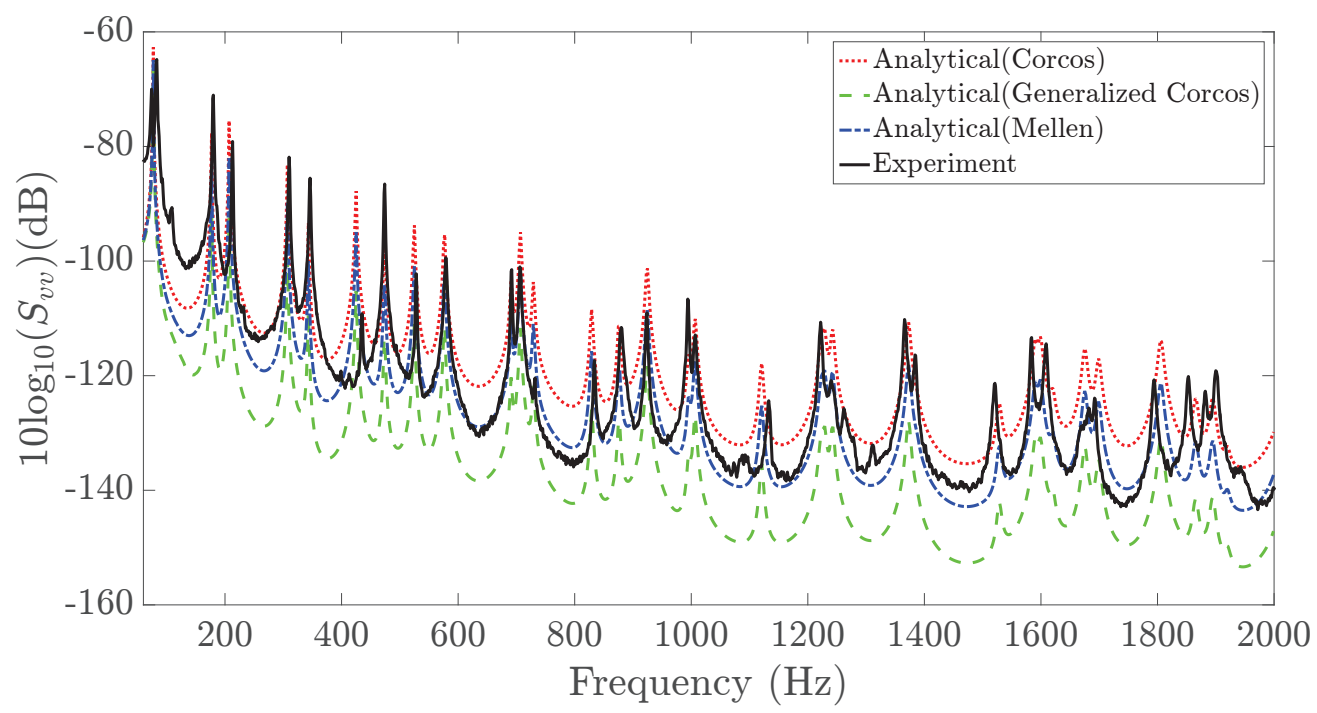

Figure 4: Predicted velocity spectra using different TBL models at flow velocity $U_{\infty}=$ $40 \mathrm{~m} / \mathrm{s}$ versus wind tunnel measurements (dB ref. $\left.1(\mathrm{~m} / \mathrm{s})^{2} / \mathrm{Hz}\right)$.

\subsubsection{Vibration response using the sensitivity function of the panel}

Figure 4 compares the velocity spectra predicted analytically with the experimental data at a flow speed of $40 \mathrm{~m} / \mathrm{s}$, for a frequency range from $60 \mathrm{~Hz}$ to $2 \mathrm{kHz}$. The Goody model described in Appendix A was used to evaluate the ASD function of the wall pressure field. Note that $\Psi_{p p}(\omega)$ is a one-sided radial frequency spectrum. Hence $\Psi_{p p}(\omega)$ was multiplied by $2 \pi$ to convert it to a one-sided cyclic frequency spectrum density $\Psi_{p p}(f)$. For the normalized CSD function, the Corcos, generalized Corcos and Mellen models described in Appendix B were employed, whereby analytical results for each model are compared. The TBL parameters were calculated based on theoretical formula for a flat plate from literature. The boundary layer thickness $\delta$ and the displacement thickness $\delta^{*}$ are given by (Çengel and Cimbala, 2006)

$$
\delta \cong \frac{0.38 x}{\operatorname{Re}_{x}^{0.2}}, \quad \delta^{*} \cong \frac{0.048 x}{\operatorname{Re}_{x}^{0.2}}
$$

where $\mathrm{Re}_{x}$ represents the Reynolds number and $x$ corresponds to the distance from the nozzle to the centre of the panel. The wall shear stress $\tau_{w}$ was calculated using the following empirical relations (Hambric et al., 2004)

$$
\tau_{w} \cong \frac{0.0225 \rho_{f} U_{\infty}^{2}}{\left(8 U_{\infty} \delta^{*} / \nu\right)^{0.25}}
$$


where $U_{\infty}$ is the free flow velocity, $\rho_{f}$ is the fluid density and $\nu$ is the kinematic viscosity. The convective velocity $U_{c}$ was approximated as follows (Bull, 1967)

$$
U_{c} \cong U_{\infty}\left(0.59+0.3 e^{-0.89 \delta^{*} \omega / U_{\infty}}\right) .
$$

It can be observed from Figure 4 that analytical results using the Mellen model are in very good agreement with experimental data. Compared to experimental results, results obtained using the Corcos model are over predicted but under predicted using the generalized Corcos model. Over-prediction of the pressure spectrum at low wavenumbers using the Corcos model has been previously reported (Graham, 1997). Discrepancy between predicted results for the three TBL models is examined in further detail in Appendix C.
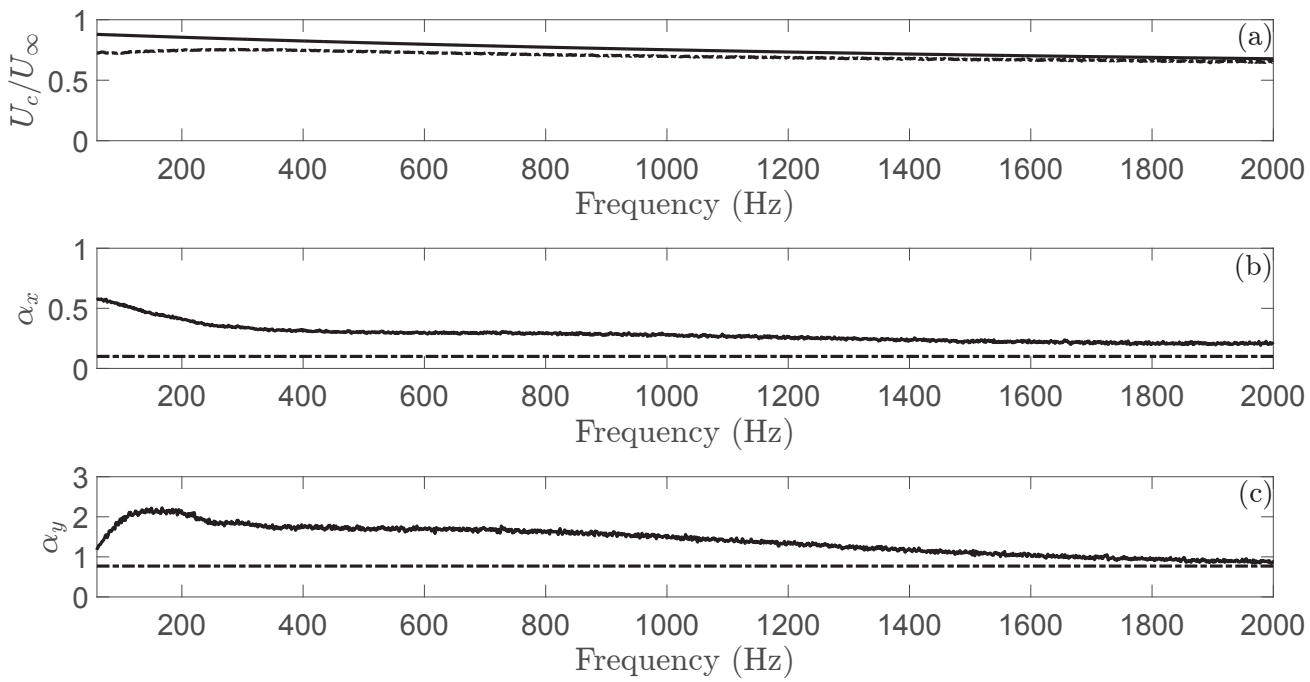

Figure 5: Comparison between TBL parameters extracted from measurements (solid line) and the standard TBL parameters from literature (dotted-dash line) at $U_{\infty}=40 \mathrm{~m} / \mathrm{s}$, (a) convective speed normalized by the flow velocity, (b) streamwise exponential decay rate $\alpha_{x}$, (c) spanwise exponential decay rate $\alpha_{y}$

The input TBL parameters for the Mellen and Goody models corresponding to the convective velocity, boundary layer thickness, displacement thickness and wall shear stress were obtained using theoretical and empirical equations from the literature given by equations (20)-(22). In the experiment, the Mellen model was fitted to the measured wall pressure field using the least square method to estimate the decay rates, $\alpha_{x}$ and $\alpha_{y}$, and the convective 
velocity $U_{c}$ (Marchetto et al., 2018). Figure 5 compares the convective speed and exponential decay coefficients as a function of frequency for the Mellen model extracted from the wind tunnel measurement with the standard values of these parameters from the literature for a flat plate. Figure 5 shows a good match between the convective speed given by equation (22) and those estimated from the experiment in the current frequency range. However, the measured values of the decay coefficients are larger than those commonly found in the literature. The experimental ASD function of the wall pressure is also presented as a function of frequency in Figure 6 and compared with predicted results by the Goody model. At low frequencies, the spectral level is under-predicted by the Goody model. However above $600 \mathrm{~Hz}$, the Goody model produces larger spectral values compared to the measured data. The maximum discrepancy occurs at very low frequency as can be observed in Figure 6 .

Figure 7 presents two analytical results for the vibration responses of the panel as well as the experimental velocity spectra as a reference solution. Analytical ${ }^{1}$ represents the vibration response of the panel when the standard Mellen and Goody models are implemented. Analytical ${ }^{2}$ corresponds to the predicted velocity spectra using the experimental ASD function of the wall pressure field and the Mellen model for the CSD function using the experimental input parameters. For the latter, the measured TBL parameters in Figure 5 were substituted into the Mellen model to evaluate the normalized CSD function, which was then used with the experimental pressure spectrum to calculate the forcing function for the panel. The two analytical results are in very good agreement with the measured data. Better agreement with the reference solution was observed at low frequencies using measured TBL input parameters $\left(\right.$ Analytical $\left.^{2}\right)$. This is due to the difference between the experimental ASD function and the Goody model as well as discrepancy between standard Mellen model and experimentally fitted Mellen model (see Appendix C.). 


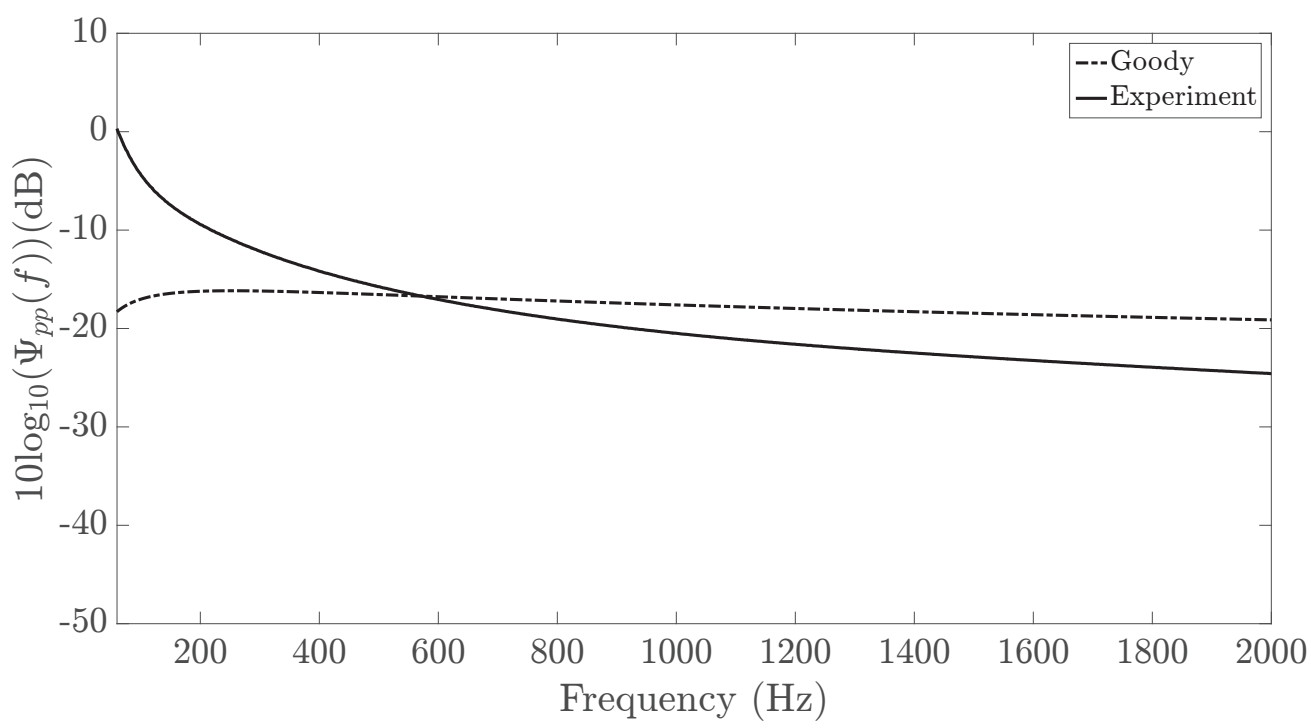

Figure 6: Measured ASD function of the wall pressure versus predicted results using the Goody model at $U_{\infty}=40 \mathrm{~m} / \mathrm{s}\left(\mathrm{dB}\right.$ ref. $\left.1 \mathrm{~Pa}^{2} / \mathrm{Hz}\right)$

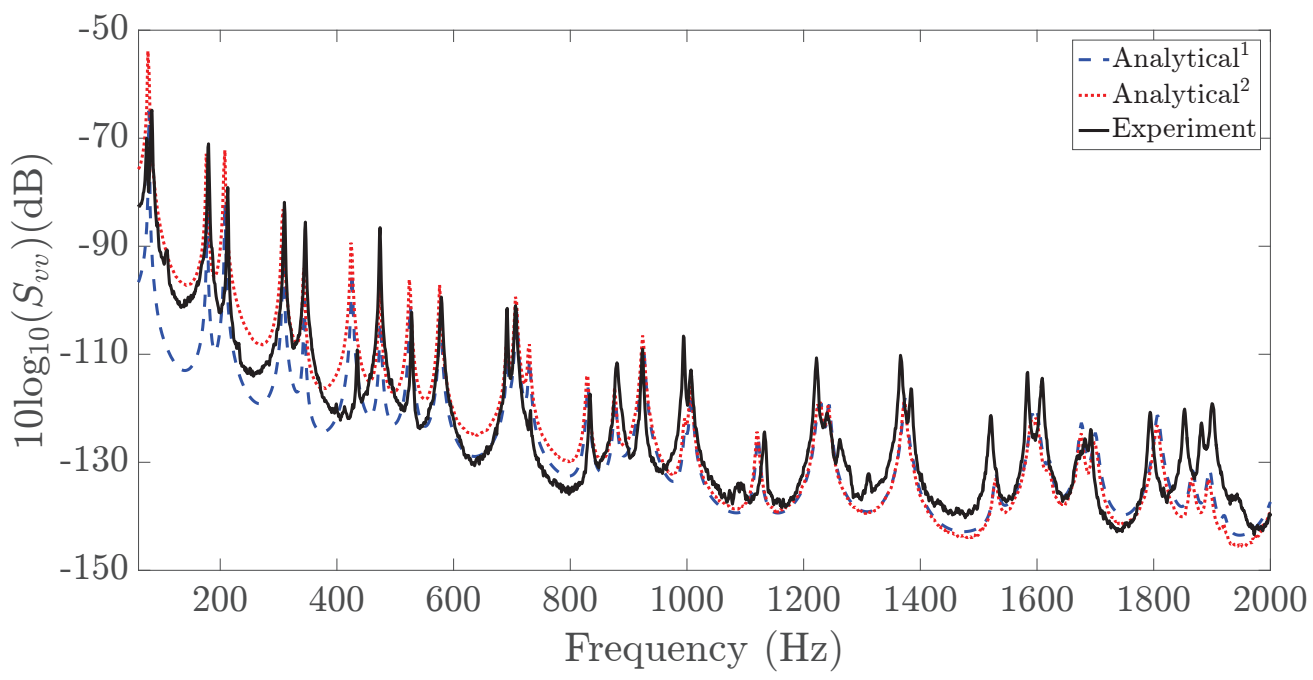

Figure 7: Velocity spectra obtained using the standard Mellen and Goody models $\left(\right.$ Analytical ${ }^{1}$ ), using the measured pressure spectrum and experimentally fitted Mellen model $\left(\right.$ Analytical $\left.{ }^{2}\right)$, and from wind tunnel measurements $\left(\mathrm{dB}\right.$ ref. $\left.1(\mathrm{~m} / \mathrm{s})^{2} / \mathrm{Hz}\right)$ 


\subsubsection{Vibration response using the hybrid UWPW-FEM approach}

The UWPW technique described in Section 2.1 was used to synthesize the pressure field at the surface of the panel. The standard Mellen and Goody models were herein used to evaluate the CSD function of the pressure field. Figure 8 shows the visualization of a realization of the surface pressure field at two discrete resonance frequencies corresponding to $177 \mathrm{~Hz}$ and $1005 \mathrm{~Hz}$, for a flow speed of $40 \mathrm{~m} / \mathrm{s}$. The Goody and Mellen models were respectively used for the ASD and normalized CSD functions of the pressure field. Figure 8(a) shows that at low frequencies, a coarse mesh can resolve the waves as they have larger wavelengths. However, at higher frequencies, a finer mesh is needed to properly describe and synthesize the wall pressure field for plane waves with short wavelengths (Figure 8(b)). In this work, the criteria used for the mesh size ensures that the plane waves with the shortest wavelength corresponding to the highest frequency of interest are adequately resolved. A mesh of 24 elements in the streamwise direction and 21 elements in the spanwise direction was used. The displacement of the panel at two discrete resonance frequencies $177 \mathrm{~Hz}$ and $1005 \mathrm{~Hz}$ are respectively shown in Figure 9 and Figure 10, using the single realization of the wall pressure given by Figures 8(a) and (b) as well as 30 realizations of the WPF. Whilst a similar pattern for displacement fields can be observed using a single realization and 30 realizations of the WPF, at least 30 realizations of the WPF are required to obtain a converged solution at the highest frequency of interest considered here. The effect of the number of realizations on the structural response of the panel is shown in Figure 11. The analytical solution described in Section 2.2 is also provided to verify the UWPW-FEM results. A close match between the numerical and analytical results can be observed. Using 30 realizations, the maximum estimated error in the calculation of the panel response was less than $1 \mathrm{~dB}$ for the frequency range considered here. As such, 30 realizations was used for all subsequent calculations.

Figure 12 presents the predicted velocity spectra using the UWPW-FEM approach as well as measured velocity spectra for the simply supported panel at flow speeds of $20 \mathrm{~m} / \mathrm{s}$ and $40 \mathrm{~m} / \mathrm{s}$. The numerical results are in excellent agreement with experimental data. As expected, with increasing flow speed, the magnitude of the vibrational response of the panel increases. A slight difference between resonant frequencies predicted numerically and obtained experimentally is observed, whereby the first ten resonances are listed in Table 2 for each case study. This can be attributed to differences in the panel 
material properties as well as in the implementation of the boundary conditions in the numerical model and the experiment. To further demonstrate capability of the UWPW-FEM approach, velocity spectra at higher flow speeds of $60 \mathrm{~m} / \mathrm{s}$ and $80 \mathrm{~m} / \mathrm{s}$ are shown in Figure 13. Numerical results show good agreement with those obtained analytically. For the parameters chosen here, the aerodynamic coincidence frequency is $7.3 \mathrm{~Hz}, 29 \mathrm{~Hz}, 66 \mathrm{~Hz}$ and $117 \mathrm{~Hz}$ for flow speeds of $20 \mathrm{~m} / \mathrm{s}, 40 \mathrm{~m} / \mathrm{s}, 60 \mathrm{~m} / \mathrm{s}$ and $80 \mathrm{~m} / \mathrm{s}$, respectively. At these frequencies, the flexural wavenumber $k_{p}=\left(\omega \sqrt{\rho_{s} h / D}\right)^{1 / 2}$ equals the convective wavenumber $k_{c}=\omega / U_{c}$ and is given by $f_{c}=U_{c}^{2} \sqrt{\rho_{s} h / D} /(2 \pi)$ and TBL strongly excites the structure (Marchetto et al., 2018).

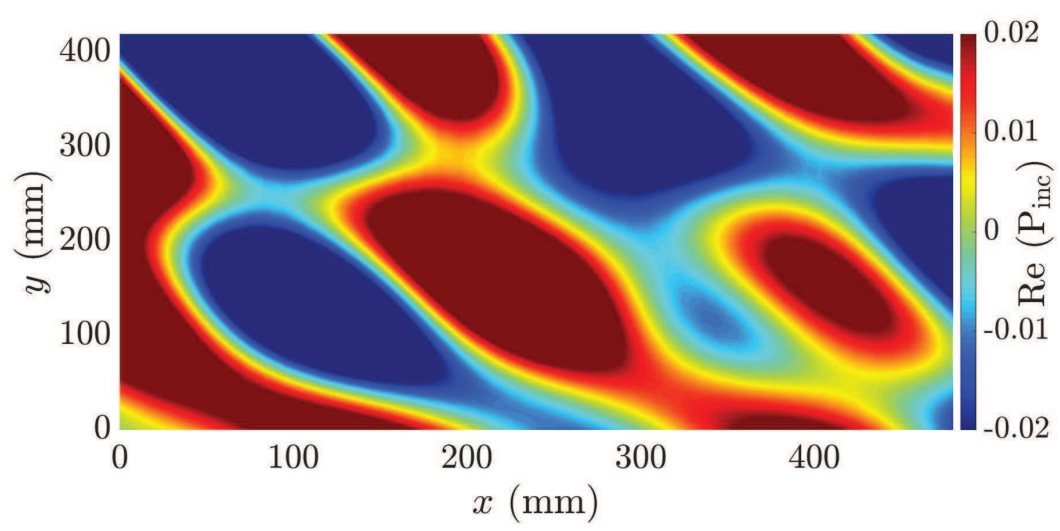

(a)

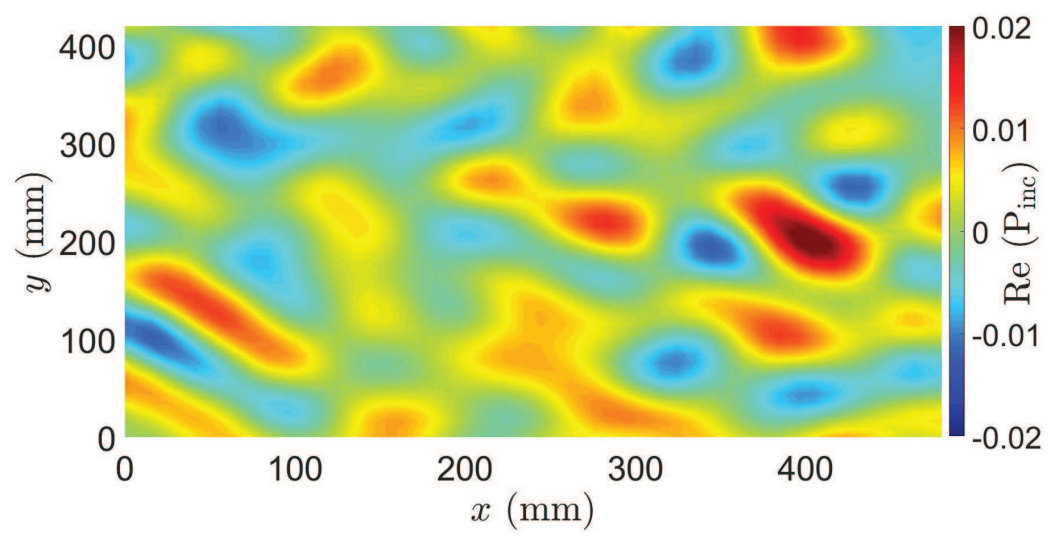

(b)

Figure 8: A realization of the wall pressure field using the Mellen and Goody models for a flow speed of $40 \mathrm{~m} / \mathrm{s}$ at (a) $177 \mathrm{~Hz}$ and (b) $1005 \mathrm{~Hz}$ 

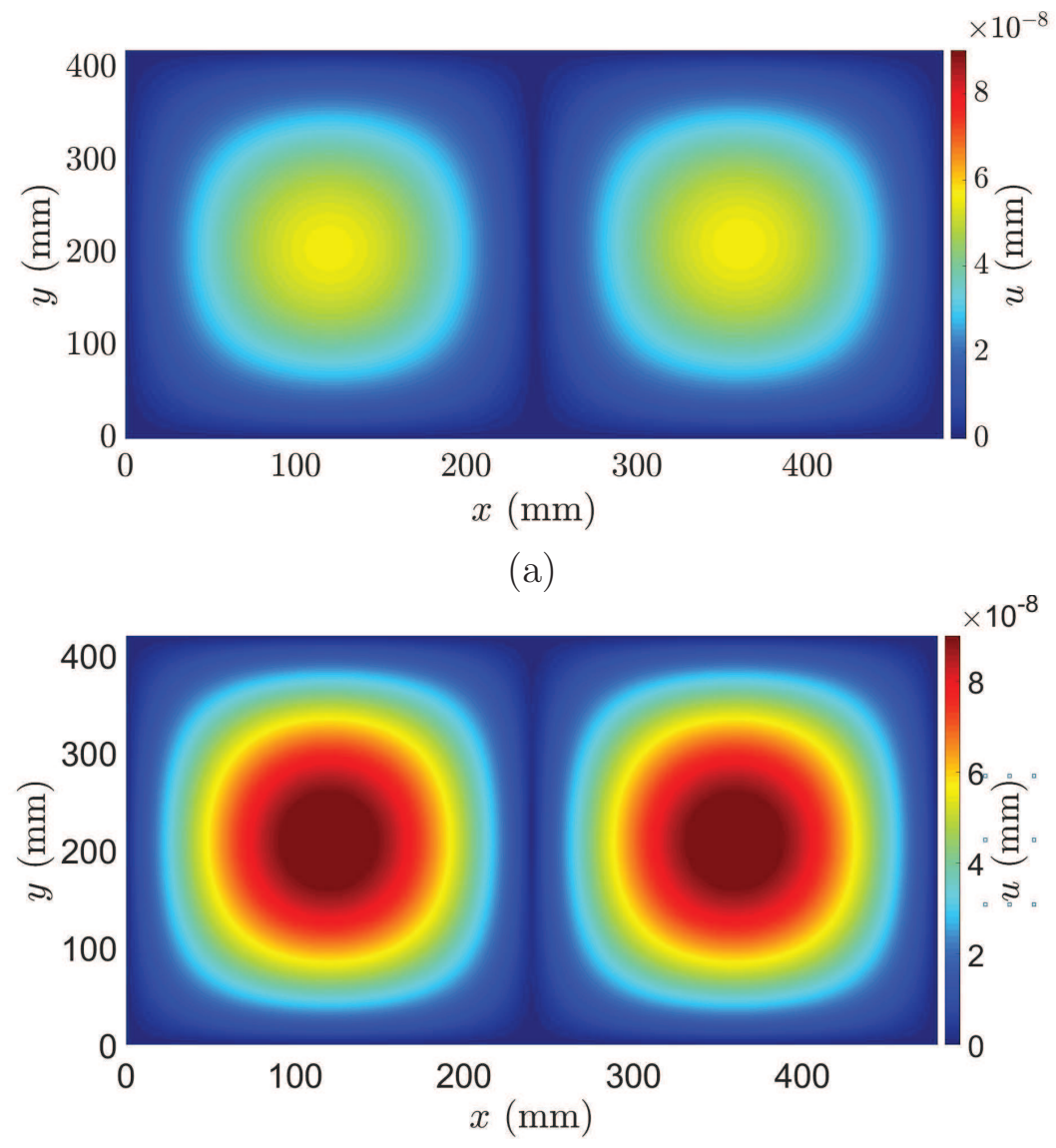

(b)

Figure 9: Panel displacement field for a flow speed of $40 \mathrm{~m} / \mathrm{s}$ at $177 \mathrm{~Hz}$ using (a) one realization of the WPF corresponding to the WPF shown in Figure 8(a) and (b) 30 realizations of the WPF 
Figure 10: Panel displacement field for a flow speed of $40 \mathrm{~m} / \mathrm{s}$ at $1005 \mathrm{~Hz}$ using (a) one realization of the WPF corresponding to the WPF shown in Figure 8(b) and (b) 30 realizations of the WPF

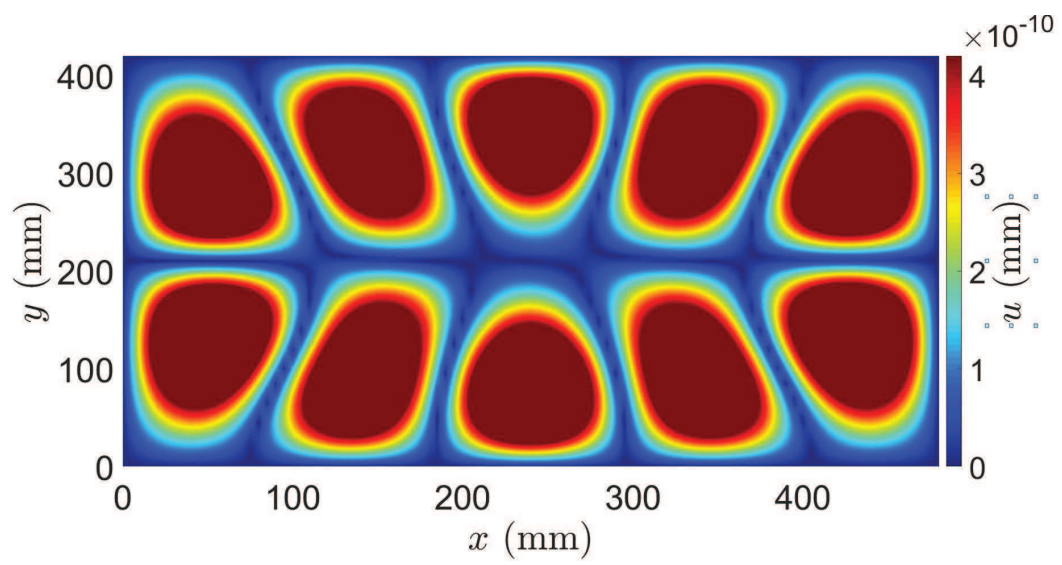

(a)

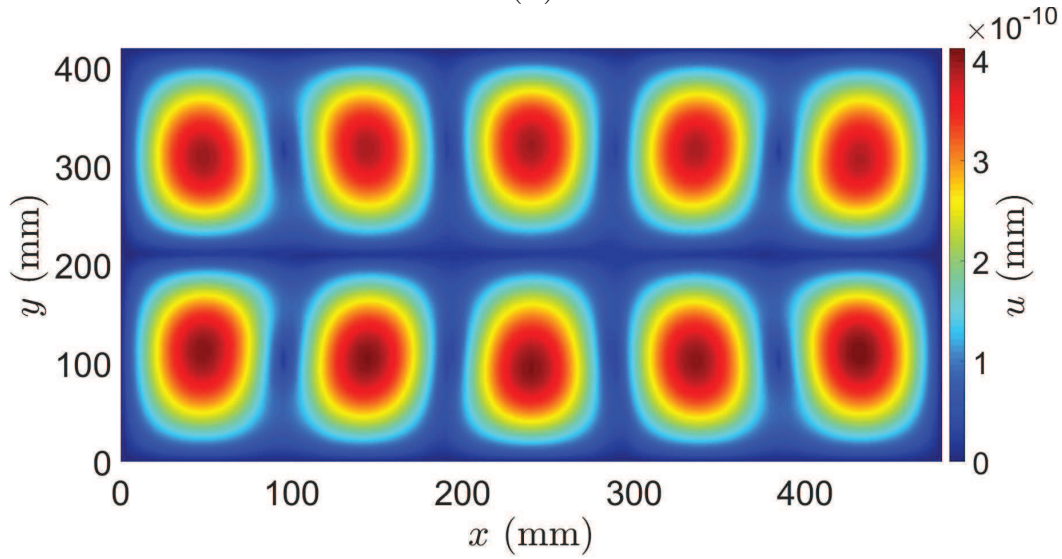

(b) 


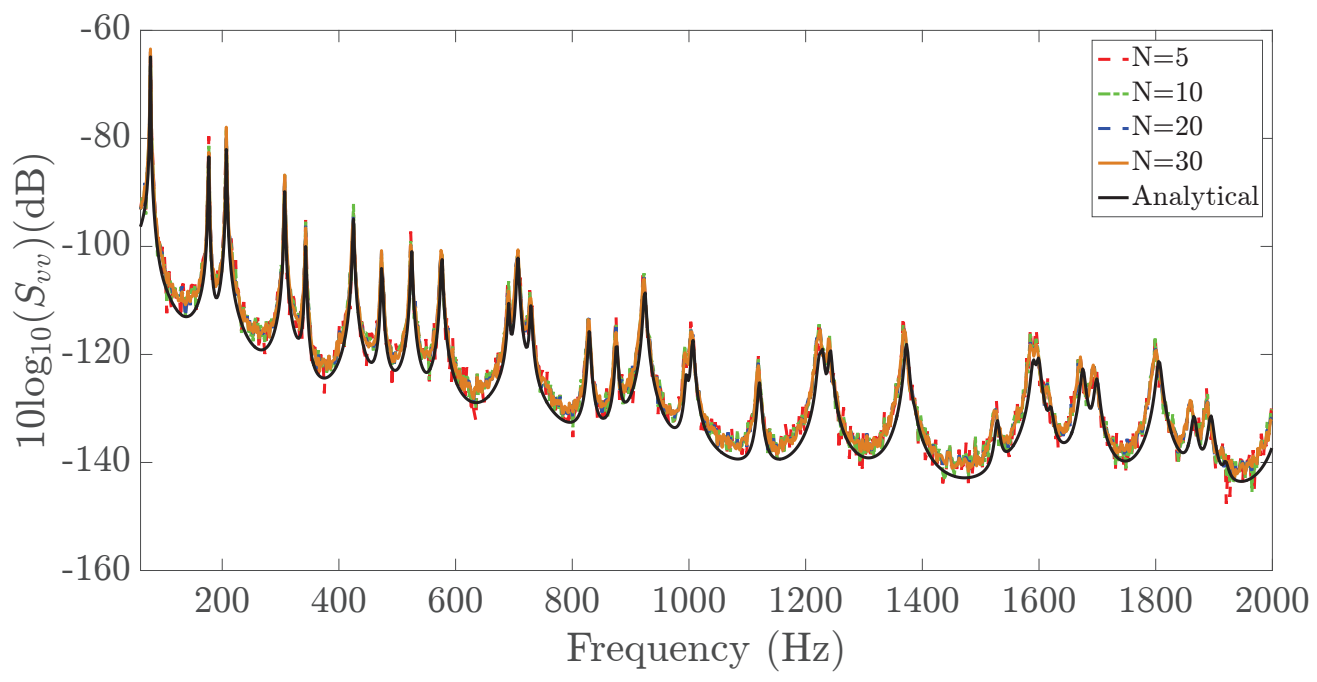

Figure 11: Velocity spectra predicted numerically using the UWPW-FEM technique for different number of realizations, as well as predicted analytically, for a flow speed of $40 \mathrm{~m} / \mathrm{s}$ $\left(\mathrm{dB}\right.$ ref. $\left.1(\mathrm{~m} / \mathrm{s})^{2} / \mathrm{Hz}\right)$ 


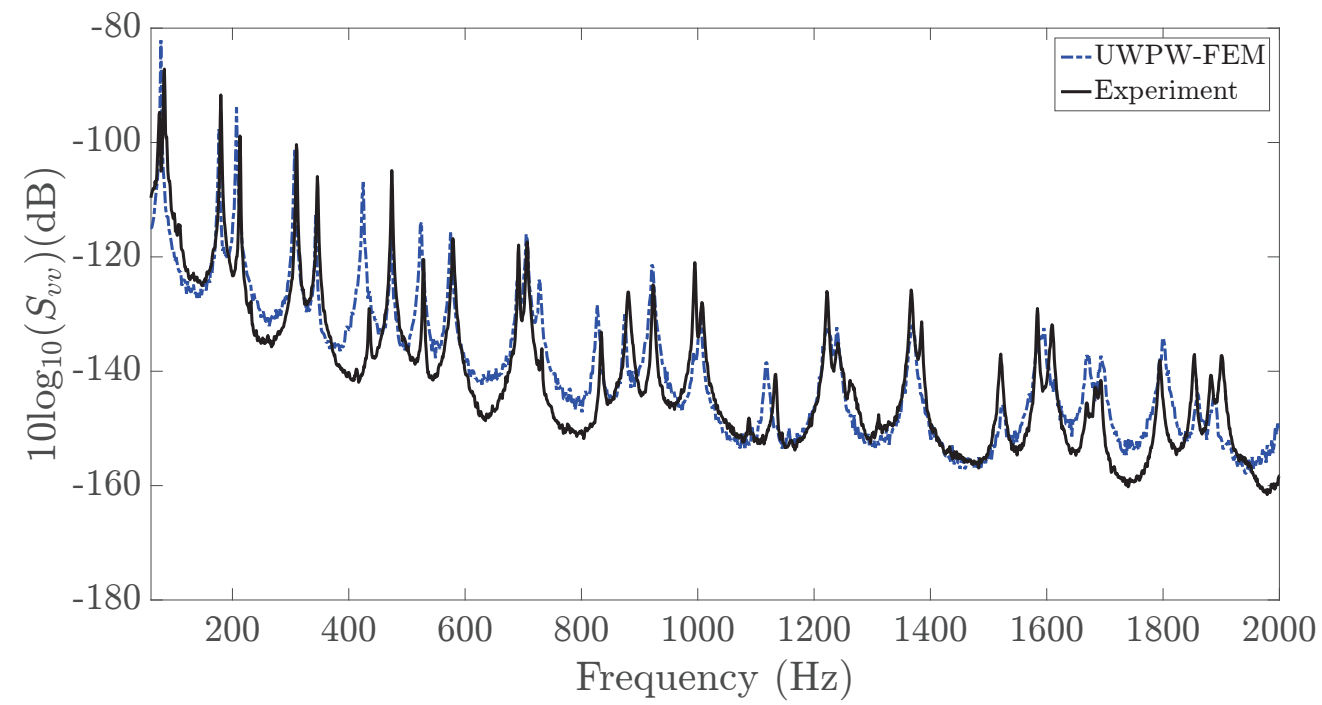

(a)

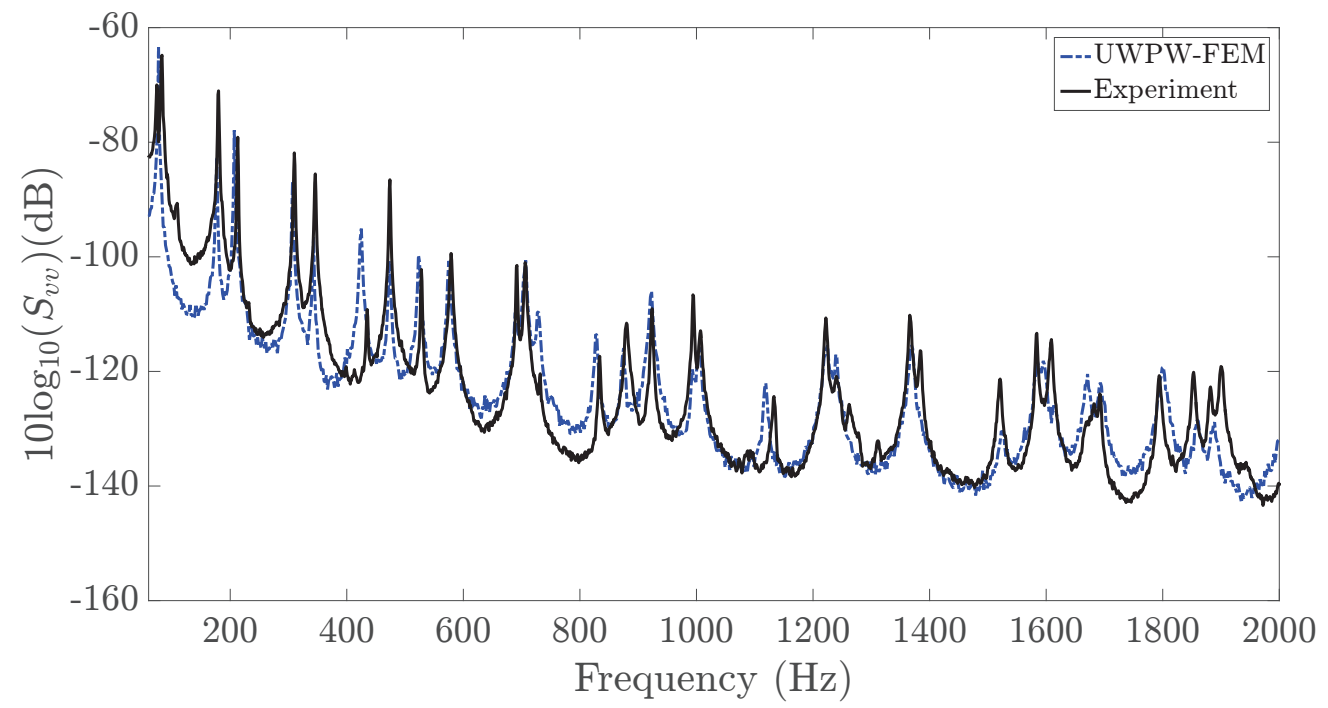

(b)

Figure 12: Predicted and measured velocity spectra for a simply supported plate for a flow speed of (a) $20 \mathrm{~m} / \mathrm{s}$ and (b) $40 \mathrm{~m} / \mathrm{s}\left(\mathrm{dB}\right.$ ref. $\left.1(\mathrm{~m} / \mathrm{s})^{2} / \mathrm{Hz}\right)$ 
Table 2. The first ten natural frequencies of the panels in case studies A and B

\begin{tabular}{lccccc}
\hline \multicolumn{3}{c}{ Case Study A } & \multicolumn{3}{c}{ Case Study B } \\
\hline Mode & FEM (Hz) & Experiment $(\mathrm{Hz})$ & Mode & FEM (Hz) & Experiment $(\mathrm{Hz})$ \\
\hline$(1,1)$ & 78 & 82 & $(1,1)$ & 84 & 86 \\
$(2,1)$ & 177 & 180 & $(2,1)$ & 144 & 148 \\
$(1,2)$ & 208 & 213 & $(1,2)$ & 192 & 192 \\
$(2,2)$ & 308 & 310 & $(3,1)$ & 244 & 244 \\
$(3,1)$ & 344 & 346 & $(2,2)$ & 248 & 246 \\
$(1,3)$ & 426 & 435 & $(3,2)$ & 344 & 345 \\
$(3,2)$ & 474 & 474 & $(1,3)$ & 358 & 356 \\
$(2,3)$ & 526 & 528 & $(4,1)$ & 380 & 378 \\
$(4,1)$ & 578 & 579 & $(2,3)$ & 412 & 389 \\
$(3,3)$ & 692 & 692 & $(4,2)$ & 476 & 413 \\
\hline
\end{tabular}

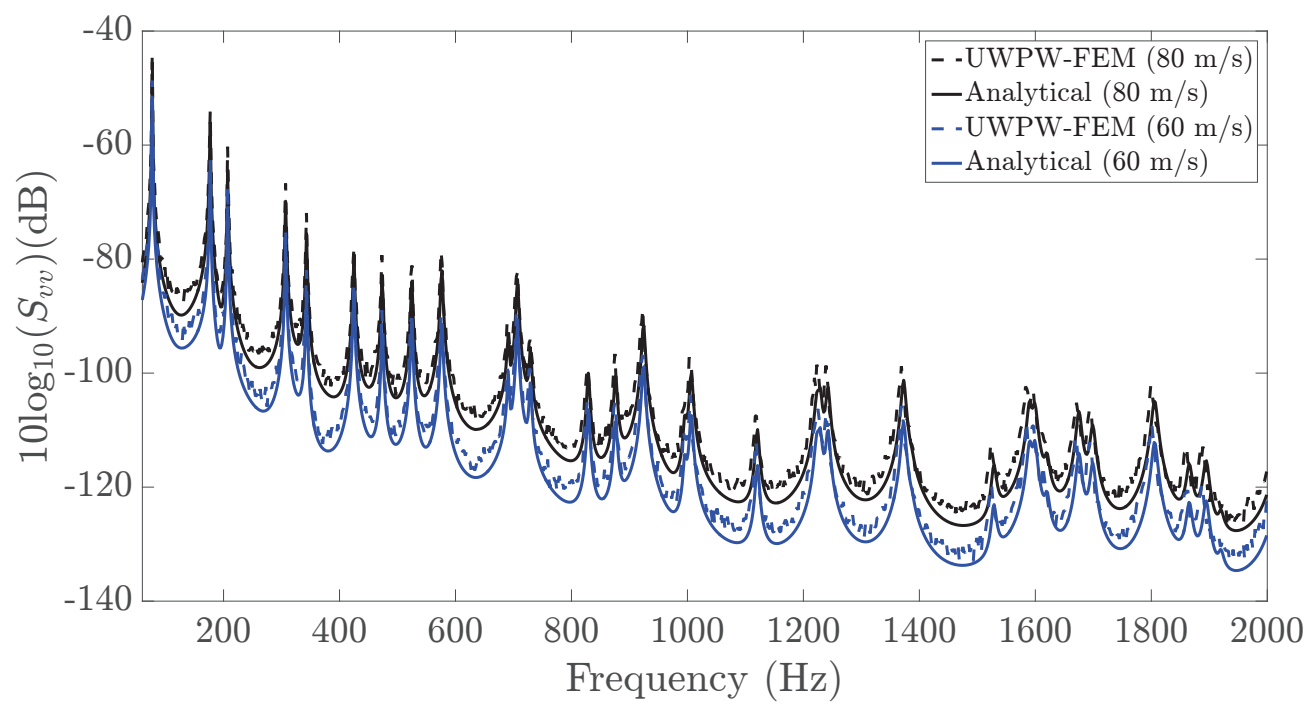

Figure 13: Predicted spectral velocity for a simply supported plate predicted numerically using the UWPW-FEM technique and analytically, for flow speeds of $60 \mathrm{~m} / \mathrm{s}$ and $80 \mathrm{~m} / \mathrm{s}$ $\left(\mathrm{dB}\right.$ ref. $\left.1(\mathrm{~m} / \mathrm{s})^{2} / \mathrm{Hz}\right)$ 


\subsection{Case study B - Clamped plate}

In the second case study we examined a steel flat plate with clamped boundary conditions along its edges excited by turbulent flow. The plate was used in the experiment conducted by Han et al. (1999) in a wind tunnel at Purdue University. Figure 14 shows a schematic diagram of the wind tunnel test section and plate. The plate vibration was measured using a scanning laser Doppler vibrometer at a point $150 \mathrm{~mm}$ from the plate's left edge and $120 \mathrm{~mm}$ from its bottom edge. The experiment was carried out at flow speeds of $35.8 \mathrm{~m} / \mathrm{s}$ and $44.7 \mathrm{~m} / \mathrm{s}$ (Han et al., 1999; Hambric et al., 2004). The displacement thickness was measured for these flow speeds to be $2.9 \mathrm{~mm}$ and $2.4 \mathrm{~mm}$, respectively. These values were used in equations (20)-(22) to calculate the input TBL parameters for the CSD function of the WPF.

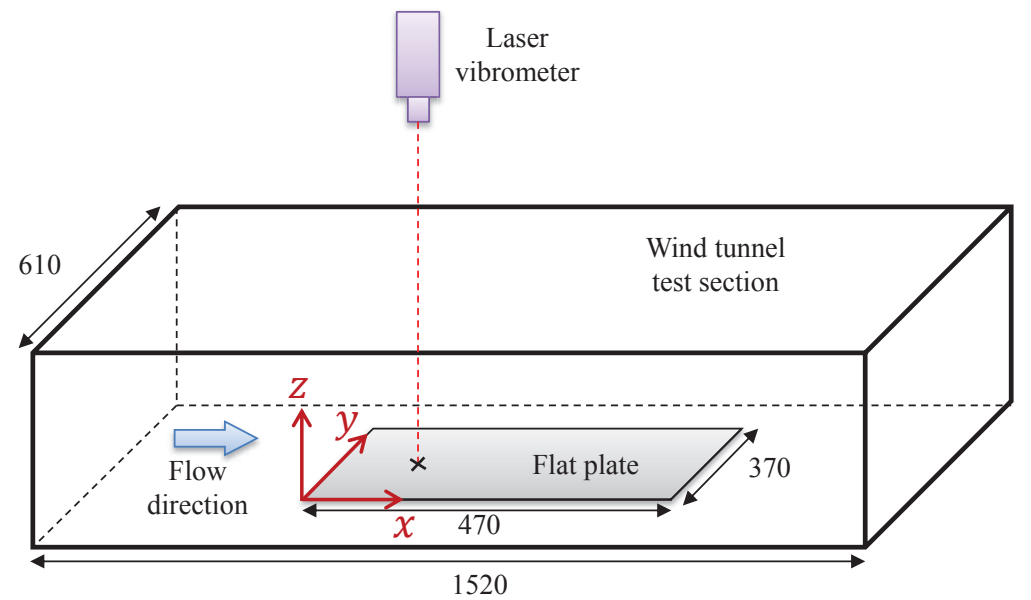

Figure 14: Schematic diagram showing the experimental set-up in the wind tunnel at Purdue University, (dimensions in $\mathrm{mm}$ )

Figure 15 presents predicted and measured velocity spectra for the clamped plate at a flow speed of $44.7 \mathrm{~m} / \mathrm{s}$. The UWPW-FEM results are shown using the Corcos, generalized Corcos and Mellen models. A similar trend to Figure 4 was observed indicating that using the Mellen model for the normalized CSD function of the pressure field provides the closest prediction to the experimental data. Similar to the first case study, there is a small discrepancy between the predicted resonant frequencies and those obtained experimentally, whereby the first ten resonances are compared in Table 2 using the 
Mellen model. In the experiment, the plate was bolted along its edges to a fixture to simulate clamped boundary conditions. However, the experimental boundary conditions may not exactly correspond to zero displacement and zero rotation along all edges of the plate, as modelled using the FEM. Further, there may be small differences in the plate material properties in the experiment and those used in the FEM model.

For the second case study, the aerodynamic coincidence frequency is $47 \mathrm{~Hz}$ and $74 \mathrm{~Hz}$ for flow speeds of $35.8 \mathrm{~m} / \mathrm{s}$ and $44.7 \mathrm{~m} / \mathrm{s}$, respectively. The first two modes of the clamped plate occur at frequencies close to the aerodynamic coincidence frequency. The three TBL models have almost the same levels of magnitude as shown at the convection peak in Figure C1. Hence, at low frequencies, the velocity spectra generated using the three TBL models are almost identical. At higher frequencies, the predicted velocity using the three models are different from each other which is consistent with the behaviour of the TBL models in the wavenumber domain in Figure C1. Comparison between predicted spectral velocity using the Mellen model and experimental data for a flow speed of $35.8 \mathrm{~m} / \mathrm{s}$ is presented in Figure 16, showing excellent agreement. It is worth noting that the maximum flow parameter $\rho_{f} U_{\infty}^{2} L_{y}^{3} / D$ for the cases studied here is approximately 2.8 for case study A at the highest considered flow speed of $80 \mathrm{~m} / \mathrm{s}$, and 1.7 for case study B at $44.7 \mathrm{~m} / \mathrm{s}$. These values for the flow parameter are well below the critical values at which static instability (divergence) or dynamic instability (flutter) occurs (Ellen, 1973; Bochkarev et al., 2016) 


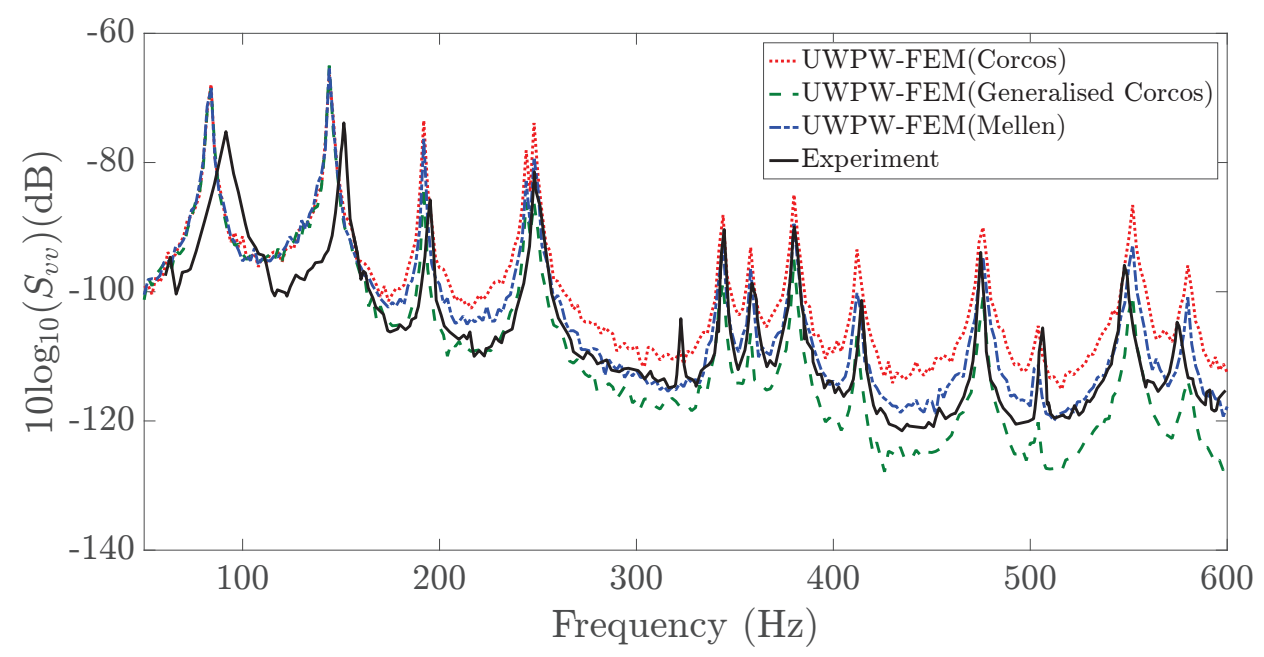

Figure 15: Predicted and measured velocity spectra for the clamped plate for a flow speed of $44.7 \mathrm{~m} / \mathrm{s}\left(\mathrm{dB}\right.$ ref. $\left.1(\mathrm{~m} / \mathrm{s})^{2} / \mathrm{Hz}\right)$

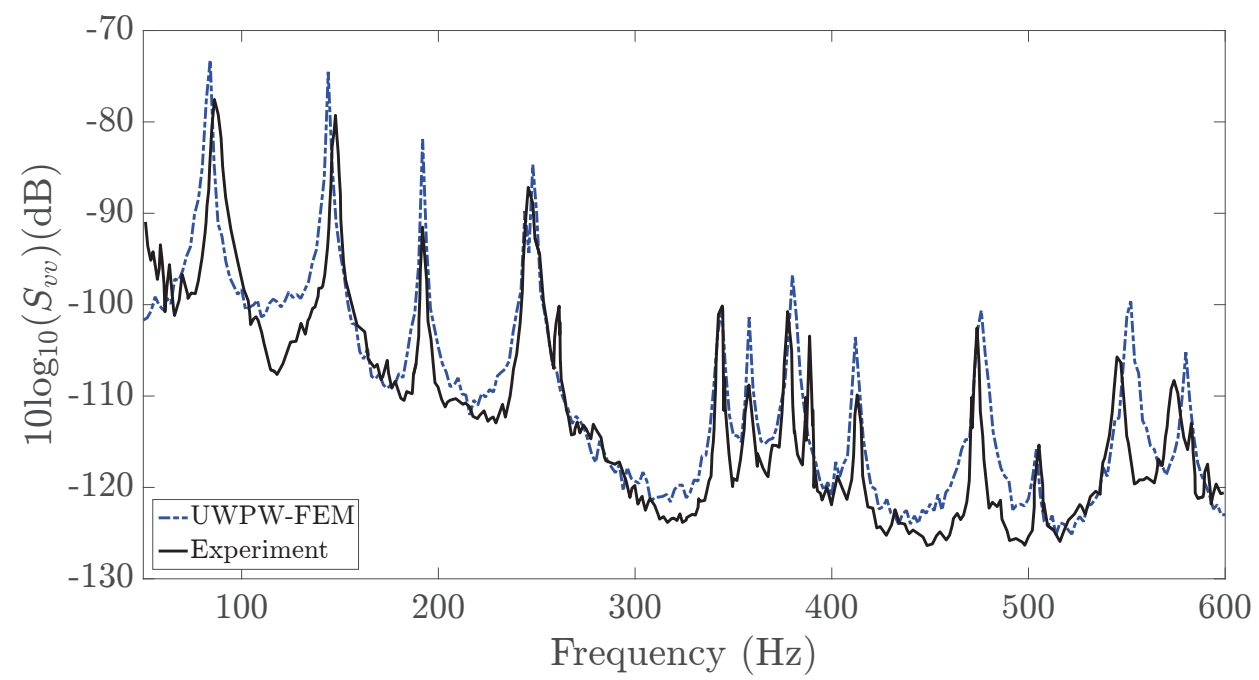

Figure 16: Predicted and measured velocity spectra for the clamped plate for a flow speed of $35.8 \mathrm{~m} / \mathrm{s}\left(\mathrm{dB}\right.$ ref. $\left.1(\mathrm{~m} / \mathrm{s})^{2} / \mathrm{Hz}\right)$. 


\section{Summary}

An uncorrelated wall plane wave technique was employed to deterministically synthesize the wall pressure field underneath a turbulent boundary layer. The pressure field was then used as an input to an FEM model of a panel to predict its vibrational response. One of the main advantages of using the UWPW technique is that the deterministic WPF is calculated at each FEM nodal point for a small number of realizations, and can be applied as an input to the FEM or any other element-based approach to evaluate the panel structural response. An analytical method based on a sensitivity function was employed to verify the numerical method for the case of a simply supported plate. Experimental data for panels with different material properties, flow conditions and boundary conditions were also used to validate the proposed approach. It was shown that the hybrid UWPW-FEM technique can be confidently used to predict the structural responses of panels excited by turbulent flow. Among the three TBL models considered in this work, the Mellen model provided better estimation of the WPF compared with experimental data. It has been shown that except at low frequencies, for a flat plate under low Mach number TBL excitation, theoretical expressions from literature can be used to estimate the input TBL parameters required to compute the CSD function of the pressure field. Whilst the case studies presented here comprise simple panels with simply supported or clamped boundary conditions, the proposed method can be applied to study the vibrations of complex panels under TBL excitation. Further, in the presence of complex flow conditions, a RANS simulation can be performed for more accurate calculation of the TBL parameters.

\section{Acknowledgements}

This research was supported by the Australian Government through the Australian Research Council's Discovery Early Career Project funding scheme (project DE190101412). 


\section{Appendices}

\section{Appendix A: Auto spectrum density function}

\section{The Goody model}

The empirical model of the auto spectrum density function of the pressure field is given by Goody (2004)

$$
\Psi_{p p}(\omega)=\frac{3 \tau_{w}^{2} \delta\left(\frac{\omega \delta}{U_{e}}\right)^{2}}{U_{e}\left(0.5+\left(\frac{\omega \delta}{U_{e}}\right)^{0.75}\right)^{3.7}\left(1.1 R_{T}^{-0.57}\left(\frac{\omega \delta}{U_{e}}\right)\right)^{7}}
$$

where $R_{T}=U_{\tau}^{2} \delta / U_{e} \nu$ and $U_{e}$ is the velocity at the boundary layer edge.

\section{Appendix B: Normalized cross spectrum density function}

The Corcos model

The Corcos normalized wavevector-frequency spectrum is given by Corcos (1963)

$$
\tilde{\phi}_{p p}\left(k_{x}, k_{y}, \omega\right)=\frac{4 \alpha_{x} \alpha_{y}}{\left(\alpha_{x}^{2}+\left(\frac{k_{x}}{k_{c}}-1\right)^{2}\right)\left(\alpha_{y}^{2}+\left(\frac{k_{y}}{k_{c}}\right)^{2}\right)}
$$

where $k_{c}=\omega / U_{c}$. The exponential decay coefficients in the normalized CSD function in the streamwise and spanwise directions are respectively $\alpha_{x}=0.1$ and $\alpha_{y}=0.77$ (Graham, 1997).

\section{The generalized Corcos model}

The normalized wavevector-frequency spectrum of the generalized Corcos model is given by Caiazzo et al. (2016)

$$
\tilde{\phi}_{p p}\left(k_{x}, k_{y}, \omega\right)=\frac{\omega^{2}}{U_{c}^{2}} \frac{4 B_{n}\left(k_{x}\right) B_{m}\left(k_{y}\right)}{\left(\frac{-A_{k_{x}} A_{k_{y}} \alpha_{\omega} \beta_{\omega}}{n m}\right) \sum_{j=0}^{n-1} e^{-\mathrm{i} \theta_{j}} \sum_{j=0}^{m-1} e^{-\mathrm{i} \theta_{j}}}
$$




$$
\begin{gathered}
B_{n}\left(k_{x}\right)=\frac{A_{k_{x}}}{1+\left(\frac{k_{x}-k_{c}}{\alpha_{\omega}}\right)^{2 n}} \\
B_{n}\left(k_{y}\right)=\frac{A_{k_{y}}}{1+\left(\frac{k_{y}}{\beta_{\omega}}\right)^{2 m}} \\
A_{k_{x}}=\frac{n \sin \left(\frac{\pi}{2 n}\right)}{\pi \alpha_{\omega}} ; \quad A_{k_{y}}=\frac{m \sin \left(\frac{\pi}{2 m}\right)}{\pi \beta_{\omega}} \\
\alpha_{\omega}=k_{c} \alpha_{x} ; \quad \beta_{\omega}=k_{c} \alpha_{y} ; \\
\theta_{j}=\frac{\pi}{2 n}(1+2 j)
\end{gathered}
$$

\section{The Mellen model}

The Mellen normalized wavenumber-frequency model is given by Mellen (1994)

$$
\tilde{\phi}_{p p}\left(k_{x}, k_{y}, \omega\right)=\frac{2 \pi\left(\alpha_{x} \alpha_{y}\right)^{2} k_{\omega}^{3}}{\left(\left(\alpha_{x} \alpha_{y} k_{c}\right)^{2}+\left(\alpha_{x} k_{y}\right)^{2}+\alpha_{y}^{2}\left(k_{x}-k_{c}\right)^{2}\right)^{3 / 2}}
$$

where $\alpha_{x}=0.1$ and $\alpha_{y}=0.77$.

\section{Appendix C}

Discrepancies in the results for the velocity spectra in Figure 4 predicted by the three TBL models are herein examined. The normalized cross spectrum of the wall pressure field as a function of non-dimensionalized wavenumber $k_{x} U_{c} / \omega$ for the three TBL models at $k_{y}=0$ are compared in Figure C1. Close to the convective peak $\left(k_{x} U_{c} / \omega=1\right)$, all three models have similar magnitudes. However, at higher frequencies or lower wavenumbers, the Corcos model provides a higher spectral level than that of the Mellen model. The generalized Corcos model with filter orders of $m=1$ and $n=2$ estimates a lower spectral level compared to the Mellen model prediction. This is consistent with the trend observed in Figure 4. It was also previously 
demonstrated that the convective peak in the Mellen model expressed in the wavenumber domain has an oval shape, which is in better agreement with the measurements, in contrast to the model of Corcos and, by extension, the generalized Corcos which has a diamond-like shape (Mellen, 1990; Miller et al., 2012).

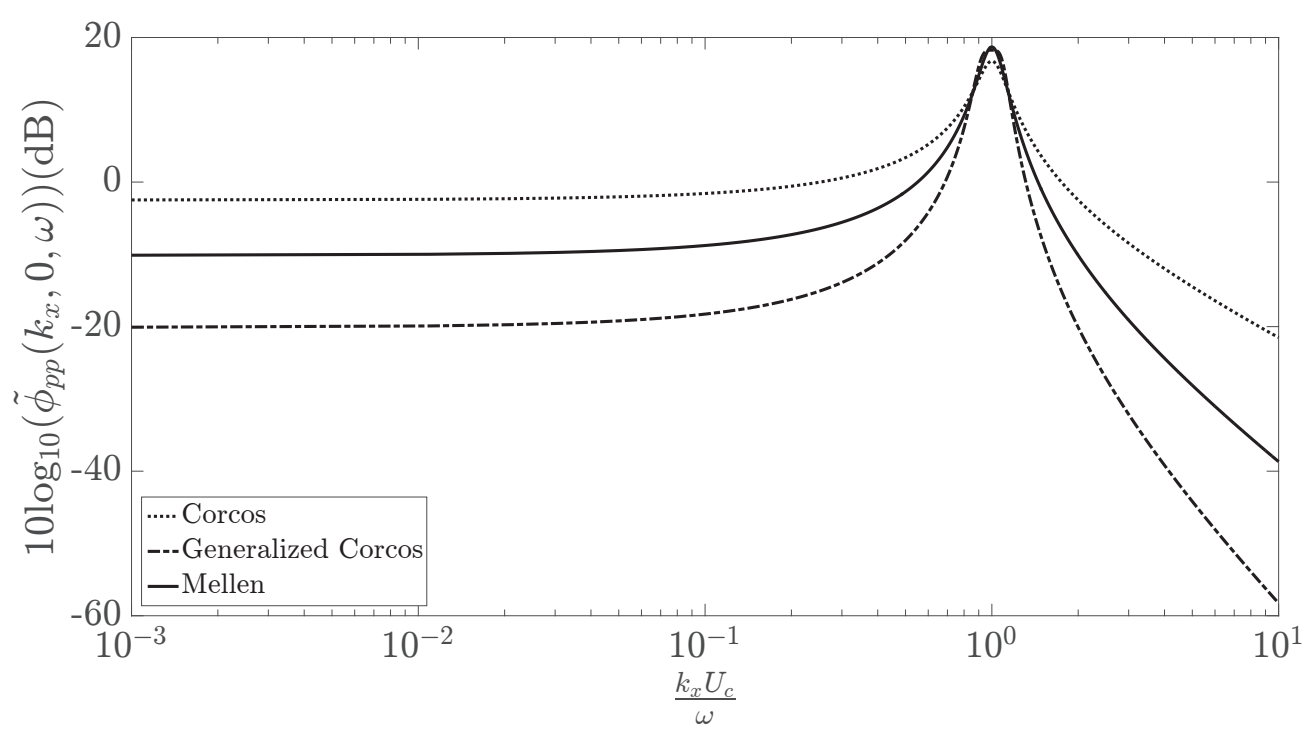

Figure C1: Normalized cross spectrum models of the wall pressure field at $k_{y}=0$

The panel filters out the pressure waves with high wavenumbers and the panel response is essentially dominated by the wavenumbers below or close to the flexural wavenumber of the panel. As such, only the wavenumber region of the normalized CSD below the plate flexural wavenumber is of interest. The normalized CSD of the wall pressure field using the Corcos model, generalized Corcos model, the standard Mellen model $\left(\right.$ Mellen $\left.^{1}\right)$ and the experimentally fitted Mellen model $\left(\right.$ Mellen $\left.^{2}\right)$ as a function of wavenumber in the streamwise direction, $k_{x}$, are compared in Figure C2, at two discrete frequencies corresponding to $200 \mathrm{~Hz}$ (Figure C2(a)) and $2000 \mathrm{~Hz}$ (Figure C2(b)). At $200 \mathrm{~Hz}$ and $2000 \mathrm{~Hz}$, the flexural wavenumbers $k_{p}$ are respectively $16(1 / \mathrm{m})$ and $51(1 / \mathrm{m})$ which are below convected wavenumber as shown in Figure C2. At $200 \mathrm{~Hz}$ (Figure C2(a)), it can be observed that the experimentally fitted Mellen model produces a higher spectral level of almost $6 \mathrm{~dB}$ than that of the standard Mellen model. Further, the experimental ASD function at $200 \mathrm{~Hz}$ 
in Figure 6 is approximately $7 \mathrm{~dB}$ above the predicted values by the Goody model. Since the CSD of the WPF is the product of the normalized CDS function and ASD function of the WPF as given by equation (1), a difference of $13 \mathrm{~dB}$ between the standard and experimental CSD functions can be observed. This explains the reason why results at $200 \mathrm{~Hz}$ in Figure 7 for the velocity spectra obtained using the standard Mellen and Goody models (Analytical ${ }^{1}$ ) are lower by almost $13 \mathrm{~dB}$ than the velocity spectra obtained using the measured pressure spectra and experimentally fitted Mellen model $\left(\right.$ Analytical $\left.^{2}\right)$. A similar interpretation can be provided for the discrepancy between the two sets of analytical results at $2000 \mathrm{~Hz}$ in Figure 7. At this frequency, the Goody model over-predicts the pressure spectrum by almost $6 \mathrm{~dB}$. Hence, when multiplied by the standard Mellen model $\left(\right.$ Mellen $\left.^{1}\right)$ which is almost $5 \mathrm{~dB}$ lower than the experimentally fitted Mellen model $\left(\right.$ Mellen $\left.^{2}\right)$, a small difference between the standard and experimental CSD functions of the wall pressure field occurs. Consequently, the velocity spectra at $2 \mathrm{kHz}$ in Figure 7 represented by Analytical ${ }^{1}$ only slightly differs from results obtained using Analytical ${ }^{2}$. 


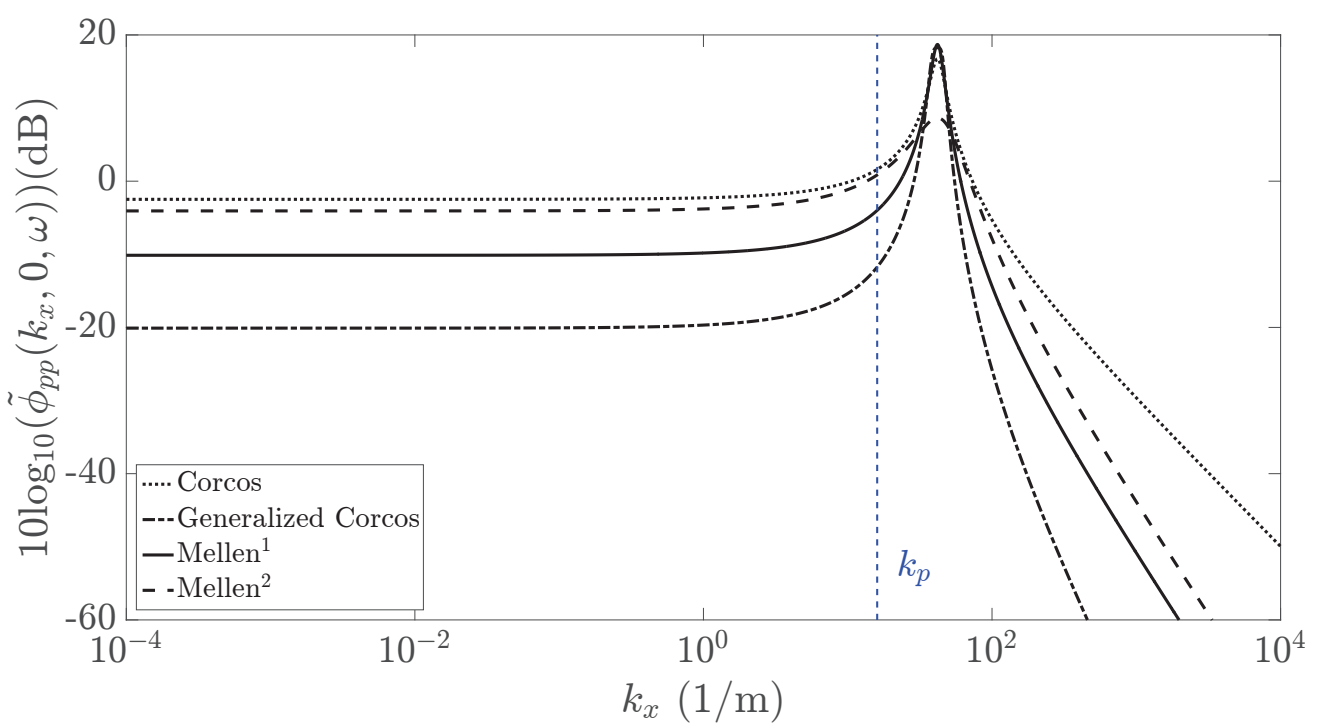

(a)

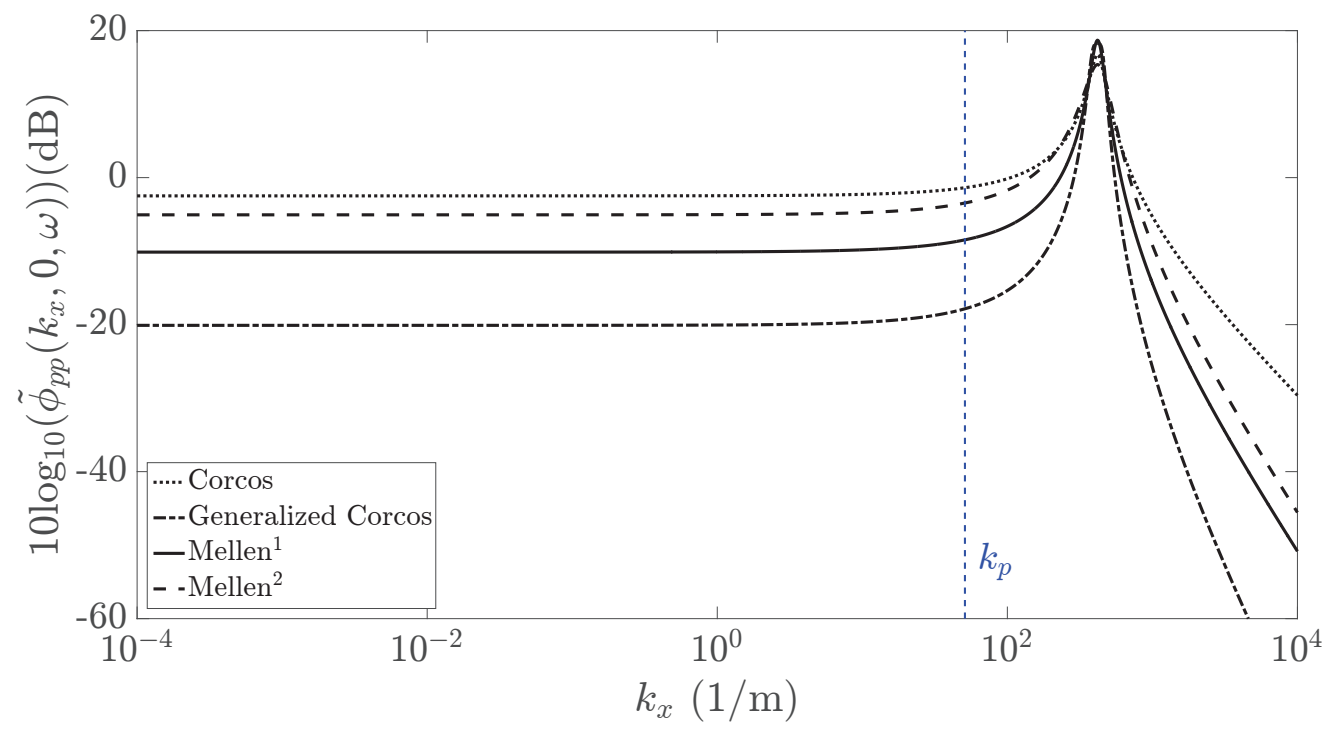

(b)

Figure C2: Normalized CSD of the wall pressure field at $k_{y}=0$ using the standard Mellen model $\left(\right.$ Mellen $\left.^{1}\right)$, the experimentally fitted Mellen model $\left(\right.$ Mellen $\left.^{2}\right)$, the Corcos model and the generalized Corcos model at (a) $200 \mathrm{~Hz}$ and (b) $2000 \mathrm{~Hz}$ (dB ref. $1 \mathrm{~Pa}^{2} / \mathrm{Hz}$ ). $k_{p}$ indicates the panel flexural wavenumber 


\section{References}

Bailly, C., Lafon, P., Candel, S., 1997. Subsonic and supersonic jet noise predictions from statistical source models. AIAA J 35, 1688-96.

Birgersson, F., Ferguson, N.S., Finnveden, S., 2003. Application of the spectral finite element method to turbulent boundary layer induced vibration of plates. J Sound Vib 259, 873-91.

Birgersson, F., Finnveden, S., 2005. A spectral super element for modelling of plate vibration. part 2: turbulence excitation. J Sound Vib 287, 315-28.

Blake, W., 1986. Mechanics of flow-induced sound and vibration: Complex flow-structure interactions. Applied Mathematics and Mechanics Series, Academic Press.

Bochkarev, S., Lekomtsev, S., Matveenko, V., 2016. Hydroelastic stability of a rectangular plate interacting with a layer of ideal flowing fluid. Fluid Dynamics 51, 821-33.

Boily, S., Charron, F., 1999. The vibroacoustic response of a cylindrical shell structure with viscoelastic and poroelastic materials. Appl Acoust 58, 131 -52 .

Bull, M., 1967. Wall-pressure fluctuations associated with subsonic turbulent boundary layer flow. J Fluid Mech 28, 719-54.

Caiazzo, A., Desmet, W., Amico, R.D., 2016. A generalized Corcos model for modelling turbulent boundary layer wall pressure fluctuations. J Sound Vib 372, 192-210.

Çengel, Y., Cimbala, J., 2006. Fluid Mechanics: Fundamentals and Applications. McGraw-Hill series in mechanical engineering, McGraw-HillHigher Education.

Chen, L., MacGillivray, I.R., 2014. Prediction of trailing edge noise based on Reynolds Averaged Navier Stokes solution. AIAA J 52, 2673-82.

Ciappi, E., De Rosa, S., Franco, F., Guyader, J., Hambric, S., 2014. Flinovia - Flow Induced Noise and Vibration Issues and Aspects: A Focus on 
Measurement, Modeling, Simulation and Reproduction of the Flow Excitation and Flow Induced Response. EBL-Schweitzer, Springer International Publishing.

Ciappi, E., De Rosa, S., Franco, F., Guyader, J., Hambric, S., Leung, R., Hanford, A., 2018. Flinovia-Flow Induced Noise and Vibration Issues and Aspects-II: A Focus on Measurement, Modeling, Simulation and Reproduction of the Flow Excitation and Flow Induced Response. Springer International Publishing.

Ciappi, E., De Rosa, S., Franco, F., Vitiello, P., Miozzi, M., 2016. On the dynamic behavior of composite panels under turbulent boundary layer excitations. J Sound Vib 364, 77-109.

Corcos, G., 1963. Resolution of pressure in turbulence. J Acoust Soc Am 35, $192-9$.

De Rosa, S., Franco, F., 2008. Exact and numerical responses of a plate under a turbulent boundary layer excitation. J Fluids Struct 24, 212-30.

De Rosa, S., Franco, F., Ciappi, E., 2015. A simplified method for the analysis of the stochastic response in discrete coordinates. J Sound Vib 339, 359-75.

Ellen, C., 1973. Stability of simply supported rectangular surfaces in uniform subsonic flow. J Appl Mech 40, Paper No. 72-APM

Errico, F., Ichchou, M., Franco, F., Rosa, S.D., Bareille, O., Droz, C., 2019. Schemes for the sound transmission of flat, curved and axisymmetric structures excited by aerodynamic and acoustic sources. J Sound Vib 456, $221-38$.

Errico, F., Ichchou, M., Rosa, S.D., Bareille, O., Franco, F., 2018. The modelling of the flow-induced vibrations of periodic flat and axial-symmetric structures with a wave-based method. J Sound Vib 424, 32-47.

Franco, F., Robin, O., Ciappi, E., Rosa, S.D., Berry, A., Petrone, G., 2019. Similitude laws for the structural response of flat plates under a turbulent boundary layer excitation. Mech Syst Signal Process 129, 590-613. 
Goody, M., 2004. Empirical spectral model of surface pressure fluctuations. AIAA J 42, 1788-93.

Graham, W., 1997. A comparison of models for the wavenumber-frequency spectrum of turbulent boundary layer pressures. J Sound Vib 206, 541-65.

Hambric, S., Hwang, Y., Bonness, W., 2004. Vibrations of plates with clamped and free edges excited by low-speed turbulent boundary layer flow. J Fluids Struct 19, 93-110.

Han, F., Bernhard, R., Mongeau, L., 1999. Prediction of flow-induced structural vibration and sound radiation using energy flow analysis. J Sound Vib 227, 685-709.

Hong, C., Shin, K.K., 2010. Modeling of wall pressure fluctuations for finite element structural analysis. J Sound Vib 329, 1673-85.

Ichchou, M., Hiverniau, B., Troclet, B., 2009. Equivalent rain on the roof loads for random spatially correlated excitations in the mid-high frequency range. J Sound Vib 322, 926-40.

Karimi, M., Croaker, P., Skvortsov, A., Moreau, D., Kessissoglou, N., 2019. Numerical prediction of turbulent boundary layer noise from a sharp-edged flat plate. Int J Numer Meth Fl 90, 522-43.

Lee, Y.T., Blake, W.K., Farabee, T.M., 2005. Modeling of wall pressure fluctuations based on time mean flow field. J Fluid Eng 127, 233-40.

Leibowitz, R., 1975. Vibroacoustic response of turbulence excited thin rectangular finite plates in heavy and light fluid media. J Sound Vib 40, 441 -95 .

Marchetto, C., Maxit, L., Robin, O., Berry, A., 2017. Vibroacoustic response of panels under diffuse acoustic field excitation from sensitivity functions and reciprocity principles. J Acoust Soc Am 141, 4508-21.

Marchetto, C., Maxit, L., Robin, O., Berry, A., 2018. Experimental prediction of the vibration response of panels under a turbulent boundary layer excitation from sensitivity functions. J Acoust Soc Am 143, 2954-64. 
Maury, C., Gardonio, P., Elliott, S., 2002. A wavenumber approach to modelling the response of a randomly excited panel, Part I: General theory. J Sound Vib 252, 83-113.

Maxit, L., 2016. Simulation of the pressure field beneath a turbulent boundary layer using realizations of uncorrelated wall plane waves. J Acoust Soc Am 140, 1268-85.

Mellen, R., 1990. On modeling convective turbulence. J Acoust Soc Am 88, 2891-3.

Mellen, R., 1994. Wave-vector filter analysis of turbulent flow. J Acoust Soc Am 95, 1671-3.

Miller, T.S., Gallman, J.M., Moeller, M.J., 2012. Review of turbulent boundary layer models for acoustic analysis. J Aircraft 49, 1739-54.

Peltier, L., Hambric, S., 2007. Estimating turbulent-boundary-layer wallpressure spectra from CFD RANS solutions. J Fluid Struct 23, 920-37.

Robin, O., Chazot, J.D., Boulandet, R., Michau, M., Berry, A., Atalla, N., 2016. A plane and thin panel with representative simply supported boundary conditions for laboratory vibroacoustic tests. Acta Acustica united with Acustica 102, 170-82.

Shtilman, L., Chasnov, J., 1992. LES versus DNS: a comparative study .

Strawderman, W.A., 1969. Turbulence-induced plate vibrations: an evaluation of finite and infinite plate models. J Acoust Soc Am 46, 1294-307. 